\title{
Effective Borel Measurability and Reducibility of Functions
}

\author{
Vasco Brattka \\ Theoretische Informatik I, FernUniversität in Hagen \\ D-58084 Hagen, Germany \\ E-Mail: vasco.brattka@fernuni-hagen.de
}

\begin{abstract}
The investigation of computational properties of discontinuous functions is an important concern in computable analysis. One method to deal with this subject is to consider effective variants of Borel measurable functions. We introduce such a notion of Borel computability for single-valued as well as for multi-valued functions by a direct effectivization of the classical definition. On Baire space the finite levels of the resulting hierarchy of functions can be characterized using a notion of reducibility for functions and corresponding complete functions. We use this classification and an effective version of a Selection Theorem of Bhattacharya-Srivastava in order to prove a generalization of the Representation Theorem of Kreitz-Weihrauch for Borel measurable functions on computable metric spaces: such functions are Borel measurable on a certain finite level, if and only if they admit a realizer on Baire space of the same quality. This Representation Theorem enables us to introduce a realizer reducibility for functions on metric spaces and we can extend the completeness result to this reducibility. Besides being very useful by itself, this reducibility leads to a new and effective proof of the Banach-Hausdorff-Lebesgue Theorem which connects Borel measurable functions with the Baire functions. Hence, for certain metric spaces the class of Borel computable functions on a certain level is exactly the class of functions which can be expressed as a limit of a pointwise convergent and computable sequence of functions of the next lower level.
\end{abstract}

Keywords: Effectively Borel measurable functions, computable analysis, effective descriptive set theory.

\section{Introduction}

Computable analysis is the theory of computability and complexity on real numbers, real number functions, and subsets of real numbers $[26,12,18]$. Often it turns out that some operation of interest is not computable and it is natural to ask for its degree of non-computability. While recursion theory [17] offers such classifications for discrete objects, we are in the domain of continuous objects and thus rather in the area of (effective) descriptive set theory [11, 15]. The purpose of this paper is to establish some links between computable analysis and effective descriptive set theory which should simplify the classification of certain operations in practice.

In the representation based approach to computable analysis $[26,5]$ Turing machines are used in order to introduce computability for functions on infinite sequences (of natural numbers or symbols) and representations are used to transfer this notion to other topological spaces of interest. Since the resulting notion of computability sensitively relies on the corresponding representations, the choice of representations is an important topic. So called admissible representations turn out to offer a good correspondence between approximation on objects and 
approximation on symbols. The Representation Theorem of Kreitz-Weihrauch [13, 26, 5] states that with respect to admissible representations a function is continuous, if and only if it admits a continuous realizer on infinite sequences (of natural numbers or symbols and with respect to the product topology). Functions which admit a computable realizer, are consequently called computable.

It is natural to ask whether this characterization can be extended to classes of Borel measurable functions. A function is called $\boldsymbol{\Sigma}_{k}^{0}$-measurable, if preimages of open sets are $\boldsymbol{\Sigma}_{k}^{0}$-sets (i.e. open sets for $k=1, F_{\sigma}$-sets for $k=2$, and so on). We call a function $\boldsymbol{\Sigma}_{k}^{0}$-computable, if these preimages can be effectively computed and it finally turns out that the Kreitz-Weihrauch Representation Theorem can be extended to these classes of functions.

We briefly sketch the organization of this paper and further results. In the next section we recall some basic notions from the representation based approach to computable analysis, such as computability with respect to representations, computable metric spaces, multi-valued operations etc. In Section 3 we recall the definitions of classes of Borel sets and classes of Borel measurable functions and we introduce representations of these classes as well as effective versions of these notions. In Section 4 we prove an effective version of the Bhattacharya-Srivastava Selection Theorem [1] which turns out to be extremely helpful in establishing our main results. For the effectivization of the classical proof multi-valued operations are employed in order to avoid non-constructive choices of points. Additionally, we discuss an effective version of the Kuratowski-Ryll-Nardzewski Selection Theorem [14] in this section which shows that Borel measurable multi-valued functions with closed images admit single-valued selectors of the same quality (from the second level on). Thus, single-valuedness is "for free" in case of computable discontinuous operations. In Section 5 we show that the classes of Borel computable functions on Baire space admit complete functions (with respect to a reducibility of functions). In Section 6 we use this completeness result together with the Bhattacharya-Srivastava Selection Theorem in order to prove an effective version of the Kreitz-Weihrauch Representation Theorem for classes of Borel measurable (and Borel computable) functions. In Section 7 we define a realizer reducibility of functions on metric spaces which allows to transfer the Completeness Theorem to functions on arbitrary computable metric spaces. In Section 8 we show that Borel measurable functions operate on inputs of a certain arithmetical complexity according to their own level of non-computability. Finally, in Section 9 we use the forementioned completeness result to prove an effective version of the Banach-Hausdorff-Lebesgue Theorem which characterizes the Borel computable functions on a certain level as pointwise limits of functions of the next lower level.

\section{Preliminaries from computable analysis}

In this section we briefly summarize some notions from computable analysis. For details the reader is referred to $[26,5]$. The basic idea of the representation based approach to computable analysis is to represent infinite objects like real numbers, functions or sets, by infinite strings of natural numbers $\mathbb{N}=\{0,1,2, \ldots\}$. Thus, a representation of a set $X$ is a surjective mapping $\delta: \subseteq \mathbb{N}^{\mathbb{N}} \rightarrow X$ and in this situation we will call $(X, \delta)$ a represented space. Here $\mathbb{N}^{\mathbb{N}}$ denotes the set of infinite sequences over $\mathbb{N}$ and the inclusion symbol is used to indicate that the mapping might be partial. If we have two represented spaces, then we can define the notion of a computable function. 
Definition 2.1 (Computable function) Let $(X, \delta)$ and $\left(Y, \delta^{\prime}\right)$ be represented spaces. A function $f: \subseteq X \rightarrow Y$ is called $\left(\delta, \delta^{\prime}\right)$-computable, if there exists some computable function $F: \subseteq \mathbb{N}^{\mathbb{N}} \rightarrow \mathbb{N}^{\mathbb{N}}$ such that $\delta^{\prime} F(p)=f \delta(p)$ for all $p \in \operatorname{dom}(f \delta)$.

The diagram in Figure 1 illustrates the situation.

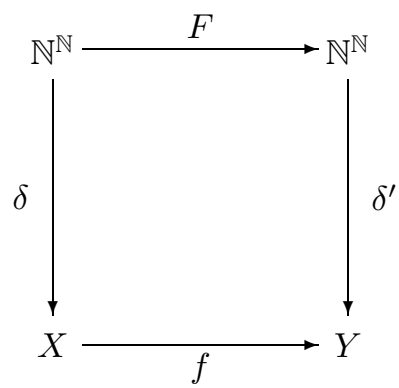

Figure 1: Computability with respect to representations

Of course, we have to define computability of functions $F: \subseteq \mathbb{N}^{\mathbb{N}} \rightarrow \mathbb{N}^{\mathbb{N}}$ to make this definition complete, but this can be done via Turing machines: $F$ is computable if there exists some Turing machine, which computes infinitely long and transforms each sequence $p$, written on the input tape, into the corresponding sequence $F(p)$, written on the one-way output tape (here we assume without loss of generality that a single position of the tape can store a natural number). We do not require natural domains for computable functions $f: \subseteq \mathbb{N}^{\mathbb{N}} \rightarrow \mathbb{N}^{\mathbb{N}}$ (which would be computable $G_{\delta}$-sets). Thus, any restriction of such a computable function is computable as well. Later on, we will also need $\left(\delta, \delta^{\prime}\right)$-computable multi-valued operations $f: \subseteq X \rightrightarrows Y$, which are defined analogously to computable functions by substituting $\delta^{\prime} F(p) \in f \delta(p)$ for the equation in Definition 2.1 above. If the represented spaces are fixed or clear from the context, then we will simply call a function or operation $f$ computable.

Analogously to the notion of computability we can define the notion of $\left(\delta, \delta^{\prime}\right)$-continuity for single- and multi-valued operations, by substituting a continuous function $F: \subseteq \mathbb{N}^{\mathbb{N}} \rightarrow \mathbb{N}^{\mathbb{N}}$ for the computable function $F$ in the definitions above. Again we will simply say that a corresponding function $f$ is continuous, if the representations are fixed or clear from the context. On $\mathbb{N}^{\mathbb{N}}$ we use the Baire topology, which is simply the product topology of the discrete topology on $\mathbb{N}$. If not mentioned otherwise, we will always assume that a represented space is endowed with the final topology induced by its representation.

For the comparison of representations it will be useful to have the notion of reducibility of representations. If $\delta, \delta^{\prime}$ are both representations of a set $X$, then $\delta$ is called reducible to $\delta^{\prime}, \delta \leq_{\mathrm{c}} \delta^{\prime}$ in symbols, if there exists a computable function $F: \subseteq \mathbb{N}^{\mathbb{N}} \rightarrow \mathbb{N}^{\mathbb{N}}$ such that $\delta(p)=\delta^{\prime} F(p)$ for all $p \in \operatorname{dom}(\delta)$. Obviously, $\delta \leq_{\mathrm{c}} \delta^{\prime}$ holds, if and only if the identity id $: X \rightarrow X$ is $\left(\delta, \delta^{\prime}\right)$-computable. Moreover, $\delta$ and $\delta^{\prime}$ are called equivalent, $\delta \equiv_{\mathrm{c}} \delta^{\prime}$ in symbols, if $\delta \leq_{\mathrm{c}} \delta^{\prime}$ and $\delta^{\prime} \leq_{\mathrm{c}} \delta$. We will use a corresponding reducibility $\leq_{\mathrm{t}}$ and equivalence $\equiv_{\mathrm{t}}$ in case that $F$ is at least continuous.

The notion of continuity with respect to representations and the ordinary notion of continuity do coincide, as long as we are dealing with admissible representations. A representation $\delta$ of a topological space $X$ is called admissible, if $\delta$ is maximal among all continuous representations $\delta^{\prime}$ of $X$, i.e. if $\delta$ is continuous and $\delta^{\prime} \leq_{\mathrm{t}} \delta$ holds for all continuous representations $\delta^{\prime}$ of $X$. If $\delta, \delta^{\prime}$ are admissible representations of $T_{0}$-spaces with countable bases, $X, Y$, then a function $f: \subseteq X \rightarrow Y$ is $\left(\delta, \delta^{\prime}\right)$-continuous, if and only if it is continuous in the ordinary topological sense. This is the classical Kreitz-Weihrauch Representation Theorem [13]. 
Given a represented space $(X, \delta)$, we will occasionally use the notions of a computable sequence and a computable point. A computable sequence is a computable function $f: \mathbb{N} \rightarrow X$, where we assume that $\mathbb{N}=\{0,1,2, \ldots\}$ is represented by $\delta_{\mathbb{N}}(p):=p(0)$ and a point $x \in X$ is called computable, if there is a constant computable function with value $x$.

Given two represented spaces $(X, \delta)$ and $\left(Y, \delta^{\prime}\right)$, there is a canonical representation $\left[\delta, \delta^{\prime}\right]$ of $X \times Y$ (defined by $\left[\delta, \delta^{\prime}\right]\langle p, q\rangle:=\left(\delta(p), \delta^{\prime}(q)\right)$ where $\langle p, q\rangle \in \mathbb{N}^{\mathbb{N}}$ denotes some standard pairing on $\mathbb{N}^{\mathbb{N}}$ ) and a representation $\left[\delta \rightarrow \delta^{\prime}\right]$ of certain functions $f: X \rightarrow Y$. If $\delta, \delta^{\prime}$ are admissible representations of $T_{0}$-spaces with countable bases, then $\left[\delta \rightarrow \delta^{\prime}\right]$ is actually a representation of the set $\mathcal{C}(X, Y)$ of continuous functions $f: X \rightarrow Y$. It can be defined via some standard representation $\eta$ of the continuous functions $F: \subseteq \mathbb{N}^{\mathbb{N}} \rightarrow \mathbb{N}^{\mathbb{N}}$ (with $G_{\delta}$-domain, see $[26,5]$ ). The function space representation can be characterized by the fact that it admits evaluation and type conversion.

Proposition 2.2 (Evaluation and type conversion) Let $(X, \delta),\left(Y, \delta^{\prime}\right)$ be admissibly represented $T_{0}$-spaces with countable bases and let $\left(Z, \delta^{\prime \prime}\right)$ be a represented space. Then:

(1) (Evaluation) ev : $\mathcal{C}(X, Y) \times X \rightarrow Y,(f, x) \mapsto f(x)$ is $\left(\left[\left[\delta \rightarrow \delta^{\prime}\right], \delta\right], \delta^{\prime}\right)$-computable,

(2) (Type conversion) $f: Z \times X \rightarrow Y$, is ([$\left.\left.\delta^{\prime \prime}, \delta\right], \delta^{\prime}\right)$-computable, if and only if the function $\check{f}: Z \rightarrow \mathcal{C}(X, Y)$, defined by $\check{f}(z)(x):=f(z, x)$ is $\left(\delta^{\prime \prime},\left[\delta \rightarrow \delta^{\prime}\right]\right)$-computable.

The proof of this proposition is based on a version of the smn- and utm-Theorem and can be found in [26]. If $(X, \delta),\left(Y, \delta^{\prime}\right)$ are admissibly represented $T_{0}$-spaces with countable bases, then in the following we will always assume that $\mathcal{C}(X, Y)$ is represented by $\left[\delta \rightarrow \delta^{\prime}\right]$. It is known that the computable points in $\left(\mathcal{C}(X, Y),\left[\delta \rightarrow \delta^{\prime}\right]\right)$ are just the $\left(\delta, \delta^{\prime}\right)$-computable functions $f: X \rightarrow Y[26]$. If $(X, \delta)$ is a represented space, then we will always assume that the set of sequences $X^{\mathbb{N}}$ is represented by $\delta^{\infty}:=\left[\delta_{\mathbb{N}} \rightarrow \delta\right]$. The computable points in $\left(X^{\mathbb{N}}, \delta^{\infty}\right)$ are just the computable sequences in $(X, \delta)$. Moreover, we assume that $X^{n}$ is always represented by $\delta^{n}$, which can be defined inductively by $\delta^{1}:=\delta$ and $\delta^{n+1}:=\left[\delta^{n}, \delta\right]$.

Now we will briefly discuss computable metric spaces, see also [5] for further details. First, we just mention that we will denote in the following the open balls of a metric space $(X, d)$ by $B(x, \varepsilon):=\{y \in X: d(x, y)<\varepsilon\}$ for all $x \in X, \varepsilon>0$ and correspondingly the closed balls by $\bar{B}(x, \varepsilon):=\{y \in X: d(x, y) \leq \varepsilon\}$. Occasionally, we denote complements of sets $A \subseteq X$ by $A^{c}:=X \backslash A$.

Definition 2.3 (Computable metric space) A tuple $(X, d, \alpha)$ is called a computable metric space, if

(1) $d: X \times X \rightarrow \mathbb{R}$ is a metric on $X$,

(2) $\alpha: \mathbb{N} \rightarrow X$ is a sequence which is dense in $X$,

(3) $d \circ(\alpha \times \alpha): \mathbb{N}^{2} \rightarrow \mathbb{R}$ is a computable (double) sequence in $\mathbb{R}$.

Here, we tacitly assume that the reader is familiar with the notion of a computable sequence of reals, but we will come back to that point below. Occasionally, we will say for short that $X$ is a computable metric space. Obviously, a computable metric space is especially separable. We will sometimes say that $(X, d, \alpha)$ is a separable metric space, if $(X, d, \alpha)$ just fulfills (1) and (2) in the definition above. 
Definition 2.4 (Cauchy representation) Let $(X, d, \alpha)$ be a computable metric space. The Cauchy representation $\delta_{X}: \subseteq \mathbb{N}^{\mathbb{N}} \rightarrow X$ of $X$ is defined by

$$
\delta_{X}(p):=\lim _{i \rightarrow \infty} \alpha p(i)
$$

for all $p$ such that $(\alpha p(i))_{i \in \mathbb{N}}$ converges and $d(\alpha p(i), \alpha p(j))<2^{-i}$ for all $j>i$ (and undefined for all other input sequences).

It is known that the representation with $\leq$ instead of $<$ is computably equivalent to $\delta_{X}$. In our context the definition above has the advantage that $\operatorname{dom}\left(\delta_{X}\right)$ is a computable metric subspace of $\mathbb{N}^{\mathbb{N}}$ (see below). In the following we tacitly assume that computable metric spaces are represented by their Cauchy representations. If $X$ is a computable metric space, then it is easy to see that $d: X \times X \rightarrow \mathbb{R}$ is computable (see Proposition 5.3 in [5]). All Cauchy representations are admissible with respect to the corresponding metric topology. If $X, Y$ are separable metric spaces and $F: \subseteq \mathbb{N}^{\mathbb{N}} \rightarrow \mathbb{N}^{\mathbb{N}}$ is a function with $\operatorname{dom}(F)=\operatorname{dom}\left(\delta_{X}\right)$ such that $\delta_{Y} F=f \delta_{X}$, then we will say that $F$ is a realizer of $f$ and we will write $F \vdash f$.

An important computable metric space is $\left(\mathbb{R}, d_{\mathbb{R}}, \alpha_{\mathbb{R}}\right)$ with the Euclidean metric $d_{\mathbb{R}}(x, y):=$ $|x-y|$ and some numbering of the rational numbers $\mathbb{Q}$, such as $\alpha_{\mathbb{R}}\langle i, j, k\rangle:=(i-j) /(k+1)$. Here, $\langle i, j\rangle:=1 / 2(i+j)(i+j+1)+j$ denotes Cantor pairs and this definition is extended inductively to finite tuples. For short we will occasionally write $\bar{k}:=\alpha_{\mathbb{R}}(k)$. In the following we assume that $\mathbb{R}$ is endowed with the Cauchy representation $\delta_{\mathbb{R}}$ induced by the computable metric space given above. This representation of $\mathbb{R}$ can also be defined, if $\left(\mathbb{R}, d_{\mathbb{R}}, \alpha_{\mathbb{R}}\right)$ just fulfills (1) and (2) of the definition above and this leads to a definition of computable real number sequences without circularity. The following example presents some typical computable metric spaces.

\section{Example 2.5 (Computable metric spaces)}

(1) $\left(\mathbb{R}^{n}, d_{\mathbb{R}^{n}}, \alpha_{\mathbb{R}^{n}}\right)$ with the Euclidean metric

$$
d_{\mathbb{R}^{n}}(x, y):=\sqrt{\sum_{i=1}^{n}\left|x_{i}-y_{i}\right|^{2}}
$$

and some standard enumeration $\alpha_{\mathbb{R}^{n}}$ of all rational points $\mathbb{Q}^{n}$ is a computable metric space.

(2) $\left(\mathcal{C}[0,1], d_{\mathcal{C}}, \alpha_{\mathcal{C}}\right)$ with the set $\mathcal{C}[0,1]$ of continuous real-valued functions $f:[0,1] \rightarrow \mathbb{R}$ and the supremum metric

$$
d_{\mathcal{C}}(f, g):=\|f-g\|:=\sup _{x \in[0,1]}|f(x)-g(x)|
$$

and some standard numbering $\alpha_{\mathcal{C}}$ of the rational polynomials $\mathbb{Q}[x]$ is a computable metric space. The computable points in this space are exactly the computable functions $f:[0,1] \rightarrow \mathbb{R}$.

(3) $\left(\mathcal{K}(X), d_{\mathcal{K}}, \alpha_{\mathcal{K}}\right)$ with the set $\mathcal{K}(X)$ of non-empty compact subsets of a computable metric space $(X, d, \alpha)$ and the Hausdorff metric

$$
d_{\mathcal{K}}(A, B):=\max \left\{\sup _{a \in A} \inf _{b \in B} d(a, b), \sup _{b \in B} \inf _{a \in A} d(a, b)\right\}
$$

and some standard numbering $\alpha_{\mathcal{K}}$ of the non-empty finite subsets of range $(\alpha)$ is a computable metric space. 
We proceed with a brief discussion of closure properties of computable metric spaces. All the mentioned closure properties do of course hold for separable metric spaces as well.

Proposition 2.6 (Subspaces, product and sequence spaces) Let $(X, d, \alpha),\left(Y, d^{\prime}, \alpha^{\prime}\right)$ be computable metric spaces, let $A \subseteq X$ and let $f: \mathbb{N} \rightarrow X$ be a computable sequence which is dense in $A$. Then the following holds:

(1) (Subspaces) The subspace $\left(A,\left.d\right|_{A \times A}, f\right)$ is a computable metric space too and $\left.\delta_{X}\right|^{A} \equiv_{{ }_{\mathrm{c}}} \delta_{A}$ holds for the corresponding Cauchy representations.

(2) (Product spaces) The product space $\left(X \times Y, d_{X \times Y}, \alpha_{X \times Y}\right)$, defined by

- $d_{X \times Y}\left((x, y),\left(x^{\prime}, y^{\prime}\right)\right):=\max \left\{d\left(x, x^{\prime}\right), d^{\prime}\left(y, y^{\prime}\right)\right\}$ and

- $\alpha_{X \times Y}\langle i, j\rangle:=\left(\alpha(i), \alpha^{\prime}(j)\right)$,

is a computable metric space too and $\delta_{X \times Y} \equiv_{\mathrm{c}}\left[\delta_{X}, \delta_{Y}\right]$ holds for the corresponding Cauchy representations.

(3) (Sequence spaces) The sequence space $\left(X^{\mathbb{N}}, d_{X^{\mathbb{N}}}, \alpha_{X^{\mathbb{N}}}\right)$, defined by

- $d_{X^{\mathbb{N}}}\left(\left(x_{n}\right),\left(y_{n}\right)\right):=\sum_{i=0}^{\infty} 2^{-i-1} \frac{d\left(x_{i}, y_{i}\right)}{1+d\left(x_{i}, y_{i}\right)}$ and

- $\alpha_{X^{\mathbb{N}}}\left\langle\left\langle n_{0}, \ldots, n_{k}\right\rangle, k\right\rangle(i):= \begin{cases}\alpha\left(n_{i}\right) & \text { if } i \leq k \\ \alpha(0) & \text { else }\end{cases}$

is a computable metric space too and $\delta_{X^{\mathbb{N}}} \equiv_{\mathrm{c}} \delta_{X}^{\infty}$ holds for the corresponding Cauchy representations.

As we have mentioned above, $\operatorname{dom}\left(\delta_{X}\right)$ is a computable metric subspace of $\mathbb{N}^{\mathbb{N}}$ for any computable metric space $X$ (since we can effectively enumerate all sequences $n_{0} n_{1} \ldots n_{k} n_{k} n_{k} \ldots \in$ $\left.\operatorname{dom}\left(\delta_{X}\right)\right)$. The proof is straightforward.

We close this section with a discussion of multi-valued functions. By $f: \subseteq X \rightrightarrows Y$ we will denote partial multi-valued functions which we will call for short operations in the following. Here the symbol "马" indicates that $f$ might be multi-valued. More precisely, an operation $f: \subseteq X \rightrightarrows Y$ is a correspondence $f=(\Phi, X, Y)$, that is $\Phi \subseteq X \times Y$. We will use these objects from an operational point of view, that is $X$ is considered as a space of inputs and $Y$ as a space of outputs. We will use some notations for operations: $\operatorname{graph}(f):=\Phi, \operatorname{dom}(f):=$ $\{x \in X:(\exists y \in Y)(x, y) \in \Phi\}$, and range $(f):=\{y \in Y:(\exists x \in X)(x, y) \in \Phi\}$ will be called graph, domain, and range of $f$, respectively. The image of $A \subseteq X$ under $f$ will be denoted by $f(A):=\{y \in Y:(\exists x \in A)(x, y) \in \Phi\}$, and the preimage of $B \subseteq Y$ by $f^{-1}(B):=\{x \in X:(\exists y \in B)(x, y) \in \Phi\}$. By $f(x):=f\{x\}=\{y \in Y:(x, y) \in \Phi\}$ we denote the image of $x$ under $f$ for each $x \in \operatorname{dom}(f)$. If $f(x)$ is single-valued, i.e. $f(x)=\{y\}$ for some $y \in Y$, then we also write $f(x)=y$, as usual for functions. With each operation $f=(\Phi, X, Y)$ we associate the inverse operation $f^{-1}=\left(\Phi^{-1}, Y, X\right)$, which is given by $\Phi^{-1}:=\{(y, x):(x, y) \in \Phi\}$. We will employ the following closure schemes of multi-valued operations (see [5] for a detailed discussion of computability properties of these schemes). 
Definition 2.7 (Closure schemes) Let $X, Y, Z$ be sets.

(1) Juxtaposition: If $f: \subseteq X \rightrightarrows Y$ and $g: \subseteq X \rightrightarrows Z$ are operations, then the juxtaposition $(f, g): \subseteq X \rightrightarrows Y \times Z$ is defined by

$$
(f, g)(x):=f(x) \times g(x)=\{(y, z): y \in f(x) \text { and } z \in g(x)\}
$$

for all $x \in \operatorname{dom}(f, g):=\operatorname{dom}(f) \cap \operatorname{dom}(g)$.

(2) Product: If $f: \subseteq X \rightrightarrows Y$ and $g: \subseteq U \rightrightarrows V$ are operations, then the product $f \times g: \subseteq X \times U \rightrightarrows Y \times V$ is defined by

$$
(f \times g)(x, u):=f(x) \times g(u)=\{(y, v): y \in f(x) \text { and } v \in g(u)\}
$$

for all $(x, u) \in \operatorname{dom}(f \times g):=\operatorname{dom}(f) \times \operatorname{dom}(g)$.

(3) Composition: If $f: \subseteq X \rightrightarrows Y$ and $g: \subseteq Y \rightrightarrows Z$ are operations, then the composition $g \circ f: \subseteq X \rightrightarrows Z$ is defined by

$$
(g \circ f)(x):=g(f(x)):=\{z:(\exists y \in f(x)) z \in g(y)\}
$$

for all $x \in \operatorname{dom}(g \circ f):=\{x: f(x) \subseteq \operatorname{dom}(g)\}$.

(4) Evaluation: If $f: \subseteq X \rightrightarrows Y^{\mathbb{N}}$ is an operation, then the evaluation $f_{*}: \subseteq X \times \mathbb{N} \rightrightarrows Y$ is defined by

$$
f_{*}(x, n):=\left\{y:\left(\exists\left(y_{k}\right)_{k \in \mathbb{N}} \in f(x)\right) y_{n}=y\right\}
$$

for all $(x, n) \in \operatorname{dom}\left(f_{*}\right):=\operatorname{dom}(f) \times \mathbb{N}$.

(5) Transposition: If $f: \subseteq X \times \mathbb{N} \rightrightarrows Y$ is an operation, then the transposition $[f]: \subseteq X \rightrightarrows Y^{\mathbb{N}}$ is defined by

$$
[f](x):=\left\{\left(y_{n}\right)_{n \in \mathbb{N}}:(\forall n) y_{n} \in f(x, n)\right\}
$$

for all $x \in \operatorname{dom}([f]):=\{x:(\forall n)(x, n) \in \operatorname{dom}(f)\}$.

(6) Exponentiation: If $f: \subseteq X \rightrightarrows Y$ is an operation, then exponentiation $f^{\mathbb{N}}: \subseteq X^{\mathbb{N}} \rightrightarrows Y^{\mathbb{N}}$ is defined by

$$
f^{\mathbb{N}}\left(\left(x_{n}\right)_{n \in \mathbb{N}}\right):=\left\{\left(y_{n}\right)_{n \in \mathbb{N}}:(\forall n) y_{n} \in f\left(x_{n}\right)\right\}
$$

for all $\left(x_{n}\right)_{n \in \mathbb{N}} \in \operatorname{dom}\left(f^{\mathbb{N}}\right):=\left\{\left(x_{n}\right)_{n \in \mathbb{N}}:(\forall n) x_{n} \in \operatorname{dom}(f)\right\}$.

\section{Borel measurable functions}

In this section we want to study Borel measurable functions of certain degrees. Therefore, we have to introduce Borel sets and their representations. If $X$ is a metric space, then we denote by $\boldsymbol{\Sigma}_{1}^{0}(X)$ the set of open subsets and by $\boldsymbol{\Pi}_{1}^{0}(X)$ the set of closed subsets of $X$. As usual, we continue inductively and we denote by $\Sigma_{k+1}^{0}(X)$ the set of subsets of $X$ which can be represented as countable union of sets from $\boldsymbol{\Pi}_{k}^{0}(X)$ for any $k \geq 1$ and by $\boldsymbol{\Pi}_{k+1}^{0}(X)$ we denote the set of subsets which can be represented as complements of sets from $\boldsymbol{\Sigma}_{k+1}^{0}(X)$. Moreover, we use the notation $\boldsymbol{\Delta}_{k}^{0}(X):=\boldsymbol{\Sigma}_{k}^{0}(X) \cap \boldsymbol{\Pi}_{k}^{0}(X)$. Thus, $\boldsymbol{\Sigma}_{2}^{0}(X)$ is the set of $F_{\sigma}$-subsets of $X$ and $\boldsymbol{\Pi}_{2}^{0}(X)$ the set of $G_{\delta}$-subsets. If $X$ is clear from the context, then we sometimes omit $(X)$ or we say for short $A$ is a $\boldsymbol{\Sigma}_{k}^{0}$-set, instead of $A \in \boldsymbol{\Sigma}_{k}^{0}(X)$. Following the inductive definition of the Borel sets we immediately obtain representations of the corresponding classes. 
Definition 3.1 (Representations of Borel sets) Let $(X, d, \alpha)$ be a separable metric space. We define representations $\delta_{\boldsymbol{\Sigma}_{k}^{0}(X)}$ of $\boldsymbol{\Sigma}_{k}^{0}(X), \delta_{\boldsymbol{\Pi}_{k}^{0}(X)}$ of $\boldsymbol{\Pi}_{k}^{0}(X)$ and $\delta_{\boldsymbol{\Delta}_{k}^{0}(X)}$ of $\boldsymbol{\Delta}_{k}^{0}(X)$ for $k \geq 1$ as follows:

$$
\begin{aligned}
& \text { - } \delta_{\boldsymbol{\Sigma}_{1}^{0}(X)}(p):={\underset{\langle i, j\rangle \in \operatorname{range}(p)}{\bigcup}} B(\alpha(i), \bar{j}), \\
& \text { - } \delta_{\boldsymbol{\Pi}_{k}^{0}(X)}(p):=X \backslash \delta_{\boldsymbol{\Sigma}_{k}^{0}(X)}(p), \\
& \text { - } \delta_{\boldsymbol{\Sigma}_{k+1}^{0}(X)}\left\langle p_{0}, p_{1}, \ldots\right\rangle:=\bigcup_{i=0}^{\infty} \delta_{\boldsymbol{\Pi}_{k}^{0}(X)}\left(p_{i}\right), \\
& \text { - } \delta_{\boldsymbol{\Delta}_{k}^{0}(X)}\langle p, q\rangle=\delta_{\boldsymbol{\Sigma}_{k}^{0}(X)}(p): \Longleftrightarrow \delta_{\boldsymbol{\Sigma}_{k}^{0}(X)}(p)=\delta_{\boldsymbol{\Pi}_{k}^{0}(X)}(q),
\end{aligned}
$$

for all $p, p_{i}, q \in \mathbb{N}^{\mathbb{N}}$.

The representation $\delta_{\boldsymbol{\Pi}_{1}^{0}}$ is equivalent to the representation $\delta_{\text {union }}$ of the closed subsets defined in [7] and the representation $\delta_{\boldsymbol{\Sigma}_{2}^{0}}$ of the $G_{\delta}$-subsets has already been considered in [23] (in case of Baire space). The computable points in $\boldsymbol{\Sigma}_{1}^{0}, \boldsymbol{\Pi}_{1}^{0}$ and $\boldsymbol{\Delta}_{1}^{0}$ are just the r.e. open, the co-r.e. closed and the decidable sets, respectively. The computable points in $\boldsymbol{\Sigma}_{2}^{0}$ and $\boldsymbol{\Pi}_{2}^{0}$ are the computable $F_{\sigma^{-}}$and $G_{\delta^{-}}$subsets, respectively. In general, we will call the corresponding computable sets computable $\boldsymbol{\Sigma}_{k}^{0}$-sets and computable $\boldsymbol{\Pi}_{k}^{0}$-sets in the context of this topic (see also [8] for these computable sets). Some closure properties of these sets are easy to establish. Here and in the following computability and continuity on the Borel classes is always understood with respect to the corresponding representations.

Proposition 3.2 (Closure properties) Let $X, Y$ be computable metric spaces. The following operations are computable (with respect to the corresponding representations) for any $k \geq 1$ :

(1) $\boldsymbol{\Sigma}_{k}^{0} \hookrightarrow \boldsymbol{\Sigma}_{k+1}^{0}, \boldsymbol{\Sigma}_{k}^{0} \hookrightarrow \Pi_{k+1}^{0}, \boldsymbol{\Pi}_{k}^{0} \hookrightarrow \boldsymbol{\Sigma}_{k+1}^{0}, \boldsymbol{\Pi}_{k}^{0} \hookrightarrow \Pi_{k+1}^{0}, A \mapsto A$ (injection)

(2) $\boldsymbol{\Sigma}_{k}^{0} \rightarrow \boldsymbol{\Pi}_{k}^{0}, \boldsymbol{\Pi}_{k}^{0} \rightarrow \boldsymbol{\Sigma}_{k}^{0}, A \mapsto A^{\mathrm{c}}:=X \backslash A$ (complement)

(3) $\boldsymbol{\Sigma}_{k}^{0} \times \boldsymbol{\Sigma}_{k}^{0} \rightarrow \boldsymbol{\Sigma}_{k}^{0}, \boldsymbol{\Pi}_{k}^{0} \times \boldsymbol{\Pi}_{k}^{0} \rightarrow \boldsymbol{\Pi}_{k}^{0},(A, B) \mapsto A \cup B$ (union)

(4) $\boldsymbol{\Sigma}_{k}^{0} \times \boldsymbol{\Sigma}_{k}^{0} \rightarrow \boldsymbol{\Sigma}_{k}^{0}, \boldsymbol{\Pi}_{k}^{0} \times \boldsymbol{\Pi}_{k}^{0} \rightarrow \boldsymbol{\Pi}_{k}^{0},(A, B) \mapsto A \cap B$ (intersection)

(5) $\left(\Sigma_{k}^{0}\right)^{\mathbb{N}} \rightarrow \Sigma_{k}^{0},\left(A_{n}\right)_{n \in \mathbb{N}} \mapsto \bigcup_{n=0}^{\infty} A_{n}$ (countable union)

(6) $\left(\boldsymbol{\Pi}_{k}^{0}\right)^{\mathbb{N}} \rightarrow \boldsymbol{\Pi}_{k}^{0},\left(A_{n}\right)_{n \in \mathbb{N}} \mapsto \bigcap_{n=0}^{\infty} A_{n}$ (countable intersection)

(7) $\boldsymbol{\Sigma}_{k}^{0}(X) \times \boldsymbol{\Sigma}_{k}^{0}(Y) \rightarrow \boldsymbol{\Sigma}_{k}^{0}(X \times Y),(A, B) \mapsto A \times B$ (product)

(8) $\left(\boldsymbol{\Pi}_{k}^{0}(X)\right)^{\mathbb{N}} \rightarrow \boldsymbol{\Pi}_{k}^{0}\left(X^{\mathbb{N}}\right),\left(A_{n}\right)_{n \in \mathbb{N}} \mapsto \times_{n=0}^{\infty} A_{n}$ (countable product)

(9) $\boldsymbol{\Sigma}_{k}^{0}(X \times \mathbb{N}) \rightarrow \boldsymbol{\Sigma}_{k}^{0}(X), A \mapsto \operatorname{pr}_{1}(A):=\{x \in X:(\exists n)(x, n) \in A\}$ (countable projection)

(10) $\boldsymbol{\Sigma}_{k}^{0}(X \times Y) \times Y \rightarrow \boldsymbol{\Sigma}_{k}^{0}(X),(A, y) \mapsto A_{y}:=\{x \in X:(x, y) \in A\}$ (section)

If not mentioned otherwise, all classes are understood with respect to $X$. If $X, Y$ are separable metric spaces, then the mentioned operations are still continuous. 
Proof. We consider the computable metric spaces $(X, d, \alpha)$ and $\left(Y, d^{\prime}, \beta\right)$.

(1) We first treat the injections $\boldsymbol{\Sigma}_{k}^{0}(X) \hookrightarrow \boldsymbol{\Sigma}_{k+1}^{0}(X)$ and $\boldsymbol{\Pi}_{k}^{0}(X) \hookrightarrow \boldsymbol{\Pi}_{k+1}^{0}(X)$ and we proceed inductively. In case of $k=1$ we obtain

$$
\bar{B}(\alpha(m), \bar{j})=X \backslash \bigcup\{B(\alpha(l), \bar{i}): l, i \in \mathbb{N} \text { and } d(\alpha(l), \alpha(m))>\bar{i}+\bar{j}\} \in \mathbf{\Pi}_{1}^{0}(X)
$$

for all $m, j \in \mathbb{N}$ and this even shows that $(\bar{B}(\alpha(m), \bar{j}))_{\langle m, j\rangle \in \mathbb{N}}$ is a computable sequence in $\boldsymbol{\Pi}_{1}^{0}(X)$. Hence, we can conclude

$$
B(\alpha(n), \bar{i})=\bigcup\{\bar{B}(\alpha(m), \bar{j}): m, j \in \mathbb{N} \text { and } d(\alpha(n), \alpha(m))+\bar{j}<\bar{i}\} \in \boldsymbol{\Sigma}_{2}^{0}(X)
$$

which implies computability of $\boldsymbol{\Sigma}_{1}^{0}(X) \hookrightarrow \boldsymbol{\Sigma}_{2}^{0}(X)$. Together with (2) this directly implies computability of the injection $\boldsymbol{\Pi}_{1}^{0}(X) \hookrightarrow \boldsymbol{\Pi}_{2}^{0}(X)$.

Now, let us assume that we have proved computability of $\boldsymbol{\Sigma}_{k}^{0}(X) \hookrightarrow \boldsymbol{\Sigma}_{k+1}^{0}(X)$ and of $\boldsymbol{\Pi}_{k}^{0}(X) \hookrightarrow \boldsymbol{\Pi}_{k+1}^{0}(X)$ for some $k \geq 1$. We have to show that the same holds for $k+1$. Since any $U \in \boldsymbol{\Sigma}_{k+1}^{0}(X)$ can be written in the form $U=\bigcup_{i=0}^{\infty} A_{i}$ with $A_{i} \in \boldsymbol{\Pi}_{k}^{0}(X)$ and $\boldsymbol{\Pi}_{k}^{0}(X) \hookrightarrow \boldsymbol{\Pi}_{k+1}^{0}(X)$ is computable, it directly follows $U \in \boldsymbol{\Sigma}_{k+2}^{0}(X)$ effectively. In other words, $\boldsymbol{\Sigma}_{k+1}^{0}(X) \hookrightarrow \boldsymbol{\Sigma}_{k+2}^{0}(X)$ is computable and thus $\boldsymbol{\Pi}_{k+1}^{0}(X) \hookrightarrow \boldsymbol{\Pi}_{k+2}^{0}(X)$ as well.

Computability of $\boldsymbol{\Sigma}_{k}^{0} \hookrightarrow \boldsymbol{\Pi}_{k+1}^{0}$ and $\boldsymbol{\Pi}_{k}^{0} \hookrightarrow \boldsymbol{\Sigma}_{k+1}^{0}$ follows directly from the definitions.

(2) Computability of the complement operations $\boldsymbol{\Sigma}_{k}^{0} \hookrightarrow \boldsymbol{\Pi}_{k}^{0}$ and $\boldsymbol{\Pi}_{k}^{0} \hookrightarrow \boldsymbol{\Sigma}_{k}^{0}$ also follows directly from the definitions.

(3), (4) Computability of the union $\boldsymbol{\Sigma}_{k}^{0} \times \boldsymbol{\Sigma}_{k}^{0} \rightarrow \boldsymbol{\Sigma}_{k}^{0}$ is obvious from the definition. Let us consider the intersection $\boldsymbol{\Sigma}_{k}^{0} \times \boldsymbol{\Sigma}_{k}^{0} \rightarrow \boldsymbol{\Sigma}_{k}^{0}$. On the one hand, we obtain

$$
B(\alpha(n), \bar{i}) \cap B(\alpha(m), \bar{j})=\bigcup\{B(\alpha(l), \bar{h}): d(\alpha(n), \alpha(l))+\bar{h}<\bar{i} \text { and } d(\alpha(m), \alpha(l))+\bar{h}<\bar{j}\}
$$

for all $n, m, i, j \in \mathbb{N}$ and, on the other hand, $\bigcup_{i=0}^{\infty} U_{i} \cap \bigcup_{j=0}^{\infty} V_{j}=\bigcup_{\langle i, j\rangle=0}^{\infty}\left(U_{i} \cap V_{j}\right)$ and thus we obtain computability of the intersection $\Sigma_{k}^{0} \times \Sigma_{k}^{0} \rightarrow \Sigma_{k}^{0}$ inductively. Using computability of the intersection and the fact that $A \cap B=\left(A^{c} \cap B^{c}\right)^{c}$ and $A \cup B=\left(A^{c} \cap B^{c}\right)^{c}$ it follows that union and intersection of type $\boldsymbol{\Pi}_{k}^{0} \times \boldsymbol{\Pi}_{k}^{0} \rightarrow \boldsymbol{\Pi}_{k}^{0}$ are computable as well.

(5), (6) Computability of the union $\left(\boldsymbol{\Sigma}_{k}^{0}\right)^{\mathbb{N}} \rightarrow \boldsymbol{\Sigma}_{k}^{0}$ is a direct consequence of the definition and computability of the intersection $\left(\boldsymbol{\Pi}_{k}^{0}\right)^{\mathbb{N}} \rightarrow \mathbf{\Pi}_{k}^{0}$ follows since $\bigcap_{i=0}^{\infty} A_{i}=\left(\bigcup_{i=0}^{\infty} A_{i}^{c}\right)^{c}$.

(7) Let $\alpha^{\prime \prime}$ be the dense sequence of the product space $X \times Y$ according to Proposition 2.6. On the one hand, we obtain

$B(\alpha(n), \bar{i}) \times B(\beta(m), \bar{j})=\bigcup\left\{B\left(\alpha^{\prime \prime}\left\langle l_{1}, l_{2}\right\rangle, \bar{h}\right): d\left(\alpha(n), \alpha\left(l_{1}\right)\right)+\bar{h}<\bar{i}\right.$ and $\left.d^{\prime}\left(\beta(m), \beta\left(l_{2}\right)\right)+\bar{h}<\bar{j}\right\}$

for all $n, m, i, j \in \mathbb{N}$ and, on the other hand, $\bigcup_{i=0}^{\infty} U_{i} \times \bigcup_{j=0}^{\infty} V_{j}=\bigcup_{\langle i, j\rangle=0}^{\infty}\left(U_{i} \times V_{j}\right)$ and thus we obtain computability of the product $\boldsymbol{\Sigma}_{k}^{0}(X) \times \boldsymbol{\Sigma}_{k}^{0}(Y) \rightarrow \boldsymbol{\Sigma}_{k}^{0}(X \times Y)$ inductively.

(8) For all $A_{i} \in \boldsymbol{\Pi}_{k}^{0}(X)$ we obtain

$$
\times_{n=0}^{\infty} A_{n}=X^{\mathbb{N}} \backslash \bigcup_{n=0}^{\infty}\left(X^{i} \times\left(X \backslash A_{i}\right) \times X^{\mathbb{N}}\right) \in \Pi_{k}^{0}\left(X^{\mathbb{N}}\right) .
$$

Thus, the countable product is computable. 
(9) Here, we consider the computable metric space $\left(\mathbb{N}, d^{\prime}, \alpha^{\prime}\right)$ with the discrete metric $d^{\prime}$ and the product space $\left(X \times \mathbb{N}, d^{\prime \prime}, \alpha^{\prime \prime}\right)$. On the one hand, we obtain $\operatorname{pr}_{1}\left(B\left(\alpha^{\prime \prime}\langle n, m\rangle, \bar{i}\right)\right)=B(\alpha(n), \bar{i})$ and on the other hand $\operatorname{pr}_{1}\left(\bigcup_{i=0}^{\infty} U_{i}\right)=\bigcup_{i=0}^{\infty} \operatorname{pr}_{1}\left(U_{i}\right)$. This implies computability of the projection $\boldsymbol{\Sigma}_{k}^{0}(X \times \mathbb{N}) \rightarrow \boldsymbol{\Sigma}_{k}^{0}(X)$.

(10) Here, we consider the product space $\left(X \times Y, d^{\prime \prime}, \alpha^{\prime \prime}\right)$. If $A=\bigcup_{i=0}^{\infty} B\left(\alpha^{\prime \prime}\left\langle n_{i}, m_{i}\right\rangle, l_{i}\right)$, then

$$
A_{y}=\bigcup\left\{B\left(\alpha\left(n_{i}\right), l_{i}\right): y \in B\left(\beta\left(m_{i}\right), l_{i}\right)\right\},
$$

which yields the proof of the case $k=1$. In general, $A=\bigcup_{i=0}^{\infty} U_{i}$ implies $A_{y}=\bigcup_{i=0}^{\infty}\left(U_{i}\right)_{y}$ which proves the general case.

The proof especially shows that the closed balls $\bar{B}(\alpha(n), \bar{i})$ are $\boldsymbol{\Pi}_{1}^{0}$-computable (in general this does not hold true for closures of open balls).

Lemma 3.3 (Closed balls) If $X$ is a computable metric space, then $(\bar{B}(\alpha(n), \bar{i}))_{\langle n, i\rangle \in \mathbb{N}}$ is a computable sequence in $\Pi_{1}^{0}(X)$.

After having settled these basic properties of Borel sets, we continue with the study of Borel measurability. A multi-valued operation $f: \subseteq X \rightrightarrows Y$ is called $\boldsymbol{\Sigma}_{k}^{0}$-measurable, if and only if preimages of open subsets are $\boldsymbol{\Sigma}_{k}^{0}$-sets relatively to $\operatorname{dom}(f)$, i.e. if for any set $U \in \boldsymbol{\Sigma}_{1}^{0}(Y)$ there exists a set $V \in \boldsymbol{\Sigma}_{k}^{0}(X)$ such that $f^{-1}(U)=V \cap \operatorname{dom}(f)$. In case that $f: X \rightrightarrows Y$ is total this corresponds to the fact that the map

$$
\Sigma_{k}^{0}\left(f^{-1}\right): \Sigma_{1}^{0}(Y) \rightarrow \Sigma_{k}^{0}(X), U \mapsto f^{-1}(U)
$$

is well-defined. Actually, it is easy to see that this map is even continuous, whenever well-defined.

Lemma 3.4 Let $X$ and $Y$ be separable metric spaces and let $f: X \rightrightarrows Y$ be $\boldsymbol{\Sigma}_{k}^{0}$-measurable for some $k \geq 1$. Then $\boldsymbol{\Sigma}_{k}^{0}\left(f^{-1}\right): \boldsymbol{\Sigma}_{1}^{0}(Y) \rightarrow \boldsymbol{\Sigma}_{k}^{0}(X), U \mapsto f^{-1}(U)$ is continuous with respect to the corresponding representations.

This follows directly from $f^{-1}\left(\bigcup_{i=0}^{\infty} A_{i}\right)=\bigcup_{i=0}^{\infty} f^{-1}\left(A_{i}\right)$. A map will be called effectively $\Sigma_{k}^{0}$-measurable if we can effectively find preimages of open subsets.

Definition 3.5 (Effective Borel measurability) Let $X, Y$ be computable metric spaces. A multi-valued map $f: \subseteq X \rightrightarrows Y$ is called effectively $\boldsymbol{\Sigma}_{k}^{0}$-measurable, or $\boldsymbol{\Sigma}_{k}^{0}$-computable for short, if there is a computable map $\Phi: \boldsymbol{\Sigma}_{1}^{0}(Y) \rightrightarrows \boldsymbol{\Sigma}_{k}^{0}(X)$ such that $f^{-1}(U)=V \cap \operatorname{dom}(f)$ for all $U \in \boldsymbol{\Sigma}_{1}^{0}(Y)$ and $V \in \Phi(U)$.

Thus, a total multi-valued operation $f: X \rightrightarrows Y$ is effectively $\boldsymbol{\Sigma}_{k}^{0}$-measurable, if the map $\boldsymbol{\Sigma}_{k}^{0}\left(f^{-1}\right): \boldsymbol{\Sigma}_{1}^{0}(Y) \rightarrow \boldsymbol{\Sigma}_{k}^{0}(X), U \mapsto f^{-1}(U)$ is computable. We directly obtain the following simple characterization of $\Sigma_{k}^{0}$-computability.

Lemma 3.6 Let $X$ and $(Y, d, \alpha)$ be computable metric spaces and let $k \geq 1$. Then an operation $f: X \rightrightarrows Y$ is $\boldsymbol{\Sigma}_{k}^{0}$-computable, if and only if $\left(f^{-1}\left(V_{i}\right)\right)_{i \in \mathbb{N}}$ is a computable sequence in $\boldsymbol{\Sigma}_{k}^{0}(X)$, whenever $\left(V_{i}\right)_{i \in \mathbb{N}}$ is a computable sequence in $\boldsymbol{\Sigma}_{1}^{0}(Y)$. In particular, this holds, if and only if $\left(f^{-1}(B(\alpha(i), \bar{j}))\right)_{\langle i, j\rangle \in \mathbb{N}}$ is a computable sequence in $\boldsymbol{\Sigma}_{k}^{0}(X)$. 
Especially, this implies that the class of $\boldsymbol{\Sigma}_{k}^{0}$-computable functions $f: X \rightarrow Y$ is exactly the class of $\Sigma_{k}^{0}$-recursive functions in the sense of Moschovakis (see the Lemma of Dellacherie 3D.1 in [15]). It is also known that the $\boldsymbol{\Sigma}_{1}^{0}$-computable single-valued functions $f: X \rightarrow Y$ are exactly the computable ones (see Theorem 6.2 in [4]). By $\boldsymbol{\Sigma}_{k}^{0}\left(X \rightrightarrows Y\right.$ ) we will denote the set of $\boldsymbol{\Sigma}_{k}^{0}$ measurable multi-valued functions $f: X \rightrightarrows Y$ and correspondingly we denote by $\Sigma_{k}^{0}(X \rightarrow Y)$ the subset of single-valued functions $f: X \rightarrow Y$. We can directly derive a representation of the set of total $\Sigma_{k}^{0}$-measurable functions.

Definition 3.7 (Representation of measurable functions) Let $X, Y$ be separable metric spaces. We define representations $\delta_{\boldsymbol{\Sigma}_{k}^{0}(X \rightrightarrows Y)}$ of $\boldsymbol{\Sigma}_{k}^{0}(X \rightrightarrows Y)$ by

$$
\delta_{\boldsymbol{\Sigma}_{k}^{0}(X \rightrightarrows Y)}(p)=f: \Longleftrightarrow\left[\delta_{\boldsymbol{\Sigma}_{1}^{0}(Y)} \rightarrow \delta_{\boldsymbol{\Sigma}_{k}^{0}(X)}\right](p)=\boldsymbol{\Sigma}_{k}^{0}\left(f^{-1}\right)
$$

for all $p \in \mathbb{N}^{\mathbb{N}}, f: X \rightrightarrows Y$ and $k \geq 1$. Let $\delta_{\boldsymbol{\Sigma}_{k}^{0}(X \rightarrow Y)}$ denote the restriction to $\boldsymbol{\Sigma}_{k}^{0}(X \rightarrow Y)$.

By the Lemma 3.4 this representation is well-defined. Sometimes it will be convenient to have a variant of this representation for partial mappings with a certain domain. Let us denote by $\left.\boldsymbol{\Sigma}_{k}^{0}(X \rightrightarrows Y)\right|_{D}$ the set of partial $\boldsymbol{\Sigma}_{k}^{0}$-measurable operations with domain $D \subseteq X$. Then we can define $\delta_{\left.\boldsymbol{\Sigma}_{k}^{0}(X \rightrightarrows Y)\right|_{D}}(p)=f$ for all $p \in \mathbb{N}^{\mathbb{N}}$ and $f: \subseteq X \rightrightarrows Y$ with $D=\operatorname{dom}(f)$, whenever there exists a function $\Phi: \boldsymbol{\Sigma}_{1}^{0}(Y) \rightrightarrows \boldsymbol{\Sigma}_{k}^{0}(X)$ such that $f^{-1}(U)=V \cap \operatorname{dom}(f)$ for all $U \in \boldsymbol{\Sigma}_{1}^{0}(Y), V \in \Phi(U)$ and $\delta_{\boldsymbol{\Sigma}_{k}^{0}(Y)} F(q) \in \Phi \delta_{\boldsymbol{\Sigma}_{1}^{0}(X)}(q)$ for all $q \in \operatorname{dom}\left(\delta_{\boldsymbol{\Sigma}_{1}^{0}(X)}\right)$ and $F:=\eta_{p}$. In other words: $p$ is a name of $f: \subseteq X \rightrightarrows Y$ with $\operatorname{dom}(f)=D$, if $p$ is the name of a function $F: \subseteq \mathbb{N}^{\mathbb{N}} \rightarrow \mathbb{N}^{\mathbb{N}}$ which "realizes" a multi-valued operation $\Phi: \boldsymbol{\Sigma}_{1}^{0}(Y) \rightrightarrows \boldsymbol{\Sigma}_{k}^{0}(X)$ which in turn describes the preimage behaviour of $f$. If $D$ is a computable metric subspace of $X$, then it is not too hard to see that $\delta_{\left.\boldsymbol{\Sigma}_{k}^{0}(X \rightrightarrows Y)\right|_{D}} \equiv_{\mathrm{c}} \delta_{\boldsymbol{\Sigma}_{k}^{0}(D \rightrightarrows Y)}$ and in such a situation we use the latter and try to avoid the former.

In the following we will establish some closure properties of (effectively) Borel measurable operations (see Definition 2.7). Continuity and computability will always be understood with respect to the above defined representations.

Proposition 3.8 (Closure properties) Let $W, X, Y$ and $Z$ be computable metric spaces. The following operations are computable for all $n, k \geq 1$ :

(1) $\boldsymbol{\Sigma}_{n}^{0}(Y \rightrightarrows Z) \times \boldsymbol{\Sigma}_{k}^{0}(X \rightarrow Y) \rightarrow \boldsymbol{\Sigma}_{n+k-1}^{0}(X \rightrightarrows Z),(g, f) \mapsto g \circ f$ (composition)

(2) $\boldsymbol{\Sigma}_{k}^{0}(X \rightrightarrows Y) \times \boldsymbol{\Sigma}_{k}^{0}(X \rightrightarrows Z) \rightarrow \boldsymbol{\Sigma}_{k}^{0}(X \rightrightarrows Y \times Z),(f, g) \mapsto(x \mapsto f(x) \times g(x))$ (juxtaposition)

(3) $\boldsymbol{\Sigma}_{k}^{0}(X \rightrightarrows Y) \times \boldsymbol{\Sigma}_{k}^{0}(W \rightrightarrows Z) \rightarrow \boldsymbol{\Sigma}_{k}^{0}(X \times W \rightrightarrows Y \times Z),(f, g) \mapsto f \times g$ (product)

(4) $\boldsymbol{\Sigma}_{k}^{0}\left(X \rightrightarrows Y^{\mathbb{N}}\right) \rightarrow \boldsymbol{\Sigma}_{k}^{0}(X \times \mathbb{N} \rightrightarrows Y), f \mapsto f_{*}$ (evaluation)

(5) $\Sigma_{k}^{0}(X \times \mathbb{N} \rightrightarrows Y) \rightarrow \Sigma_{k}^{0}\left(X \rightrightarrows Y^{\mathbb{N}}\right), f \mapsto[f]$ (transposition)

(6) $\boldsymbol{\Sigma}_{k}^{0}(X \rightrightarrows Y) \rightarrow \boldsymbol{\Sigma}_{k}^{0}\left(X^{\mathbb{N}} \rightrightarrows Y^{\mathbb{N}}\right), f \mapsto f^{\mathbb{N}}$ (exponentiation)

(7) $\Sigma_{k}^{0}(X \times \mathbb{N} \rightrightarrows Y) \rightarrow \Sigma_{k}^{0}(X \rightrightarrows Y)^{\mathbb{N}}, f \mapsto(n \mapsto(x \mapsto f(x, n)))$ (sequencing)

(8) $\boldsymbol{\Sigma}_{k}^{0}(X \rightrightarrows Y)^{\mathbb{N}} \rightarrow \boldsymbol{\Sigma}_{k}^{0}(X \times \mathbb{N} \rightrightarrows Y),\left(f_{n}\right)_{n \in \mathbb{N}} \mapsto\left((x, n) \mapsto f_{n}(x)\right)$ (de-sequencing)

If $W, X, Y$ and $Z$ are separable metric spaces, then the mentioned operations are still continuous. In case of $n=1$ composition can also be extended to $\Sigma_{1}^{0}(Y \rightrightarrows Z) \times \Sigma_{k}^{0}(X \rightrightarrows Y) \rightarrow \Sigma_{k}^{0}(X \rightrightarrows Z)$. 
Proof. We tacitly apply the closure properties from Propositions 2.2 and 3.2.

(1) For all $U \in \boldsymbol{\Sigma}_{1}^{0}(Z)$ and $A_{i} \in \mathbf{\Pi}_{n-1}^{0}(Y)$ with $g^{-1}(U)=\bigcup_{i=0}^{\infty} A_{i}$ we obtain in case $n>1$

(a) $(g \circ f)^{-1}(U)=f^{-1} g^{-1}(U)$,

(b) $f^{-1}\left(\bigcup_{i=0}^{\infty} A_{i}\right)=\bigcup_{i=0}^{\infty} f^{-1}\left(A_{i}\right)$,

(c) $f^{-1}\left(A_{i}\right)=X \backslash f^{-1}\left(Y \backslash A_{i}\right)$.

Since $f^{-1}\left(Y \backslash A_{i}\right) \in \Sigma_{n+k-2}^{0}(X)$ we obtain $(g \circ f)^{-1}(U)=\bigcup_{i=0}^{\infty} f^{-1}\left(A_{i}\right) \in \Sigma_{n+k-1}^{0}(X)$.

(2) For all $U \in \boldsymbol{\Sigma}_{1}^{0}(Y)$ and $V \in \boldsymbol{\Sigma}_{1}^{0}(Z)$ we obtain $(f, g)^{-1}(U \times V)=f^{-1}(U) \cap g^{-1}(V)$.

(3) For all $U \in \boldsymbol{\Sigma}_{1}^{0}(Y)$ and $V \in \boldsymbol{\Sigma}_{1}^{0}(Z)$ we obtain $(f \times g)^{-1}(U \times V)=f^{-1}(U) \times g^{-1}(V)$.

(4) For all $U \in \boldsymbol{\Sigma}_{1}^{0}(Y)$ we obtain

$$
\left(f_{*}\right)^{-1}(U)=\bigcup_{n=0}^{\infty}\left(f^{-1}\left(Y^{n} \times U \times Y \times Y \times \ldots\right) \times\{n\}\right) .
$$

(5) For all sets $U_{0}, U_{1}, \ldots, U_{n} \in \boldsymbol{\Sigma}_{1}^{0}(Y)$ and $U:=U_{0} \times U_{1} \times \ldots \times U_{n} \times Y \times Y \times \ldots$ we obtain

$$
[f]^{-1}(U)=\operatorname{pr}_{1}\left(\bigcup_{i=0}^{n}\left(f^{-1}\left(U_{i}\right) \cap(X \times\{i\})\right)\right) .
$$

(6) For all sets $U_{0}, U_{1}, \ldots, U_{n} \in \boldsymbol{\Sigma}_{1}^{0}(Y)$ and $U:=U_{0} \times U_{1} \times \ldots \times U_{n} \times Y \times Y \times \ldots$ we obtain

$$
\left(f^{\mathbb{N}}\right)^{-1}(U)=f^{-1}\left(U_{0}\right) \times f^{-1}\left(U_{1}\right) \times \ldots \times f^{-1}\left(U_{n}\right) \times X \times X \times \ldots
$$

(7) Let $\left(f_{i}\right)_{i \in \mathbb{N}}$ be the result of the sequencing operation applied to $f$. For all $U \in \Sigma_{1}^{0}(Y)$ we obtain $f_{i}^{-1}(U)=\left(f^{-1}(U)\right)_{i}$ where ()$_{i}$ denotes the section.

(8) Let deseq : $\boldsymbol{\Sigma}_{k}^{0}(X \rightrightarrows Y)^{\mathbb{N}} \rightarrow \boldsymbol{\Sigma}_{k}^{0}(X \times \mathbb{N} \rightrightarrows Y)$ denote the operation defined in (8). For all $U \in \boldsymbol{\Sigma}_{1}^{0}(Y)$ we obtain deseq $\left(\left(f_{n}\right)_{n \in \mathbb{N}}\right)^{-1}(U)=\bigcup_{n=0}^{\infty}\left(f_{n}^{-1}(U) \times\{n\}\right)$.

The reader should observe that composition is in general only considered in those cases where the inner function $f$ is single-valued. The proof would not go trough for multi-valued $f$ since in this case property (1)(c) does not hold true in general. We could formulate non-uniform versions of the above closure properties as corollaries and as an example we formulate this for the composition. Strictly speaking, it is only a corollary in the case of total functions; however, the partial case can be proved similarly as (1) in the previous proposition.

Corollary 3.9 Let $X, Y$ and $Z$ be computable metric spaces and $n, k \in \mathbb{N}$. If $f: \subseteq X \rightarrow Y$ is $\boldsymbol{\Sigma}_{n+1}^{0}$-computable and $g: \subseteq Y \rightrightarrows Z$ is $\boldsymbol{\Sigma}_{k+1}^{0}$-computable, then $g \circ f$ is $\boldsymbol{\Sigma}_{n+k+1}^{0}$-computable.

Again, in case $n=0$ the same holds true for multi-valued functions $f: \subseteq X \rightrightarrows Y$ as well. In the following proposition we will show that a uniformly and effectively converging sequence of $\boldsymbol{\Sigma}_{k}^{0}$-computable functions converges to a $\boldsymbol{\Sigma}_{k}^{0}$-computable function. 
Proposition 3.10 (Uniform convergence) Let $X, Y$ be computable metric spaces and $k \geq 1$. The following operation is computable:

$$
\operatorname{Lim}: \subseteq \Sigma_{k}^{0}(X \rightrightarrows Y)^{\mathbb{N}} \rightarrow \Sigma_{k}^{0}(X \rightarrow Y),\left(f_{n}\right)_{n \in \mathbb{N}} \mapsto\left(x \mapsto\left\{\lim _{n \rightarrow \infty} y_{n}: y_{n} \in f_{n}(x)\right\}\right),
$$

defined for all sequences $\left(f_{n}\right)_{n \in \mathbb{N}}$ of $\boldsymbol{\Sigma}_{k}^{0}$-measurable multi-valued functions $f_{n}: X \rightrightarrows Y$ which fulfill $d\left(y_{i}, y_{j}\right)<2^{-j}$ for all $x \in X$ and $i>j$ where $y_{n} \in f_{n}(x)$ and any such sequence $\left(y_{n}\right)_{n \in \mathbb{N}}$ is convergent.

Proof. Let $\operatorname{Lim}_{Y}: \subseteq Y^{\mathbb{N}} \rightarrow Y$ be the limit operation which is defined for all convergent sequences $\left(y_{n}\right)_{n \in \mathbb{N}}$ such that $d\left(y_{i}, y_{j}\right)<2^{-j}$ for all $i>j$ and which is computable (see Proposition 5.3 in [5]) and let deseq denote the de-sequencing operation. Then we obtain $\operatorname{Lim}\left(f_{n}\right)_{n \in \mathbb{N}}=$ $\operatorname{Lim}_{Y} \circ\left[\operatorname{deseq}\left(\left(f_{n}\right)_{n \in \mathbb{N}}\right)\right]$, i.e. Lim can be obtained with the help of de-sequencing, transposition and composition and hence Lim is computable by Proposition 3.8.

We directly formulate a non-uniform corollary for the case of single-valued functions.

Corollary 3.11 Let $X, Y$ be computable metric spaces and $k \geq 1$. If $\left(f_{n}\right)_{n \in \mathbb{N}}$ is a computable and pointwise convergent sequence of $\boldsymbol{\Sigma}_{k}^{0}$-computable functions $f_{n}: X \rightarrow Y$ such that additionally $d\left(f_{i}(x), f_{j}(x)\right)<2^{-j}$ for all $x \in X$ and $i>j$, then the limit function $f: X \rightarrow Y$ is $\Sigma_{k}^{0}$-computable as well.

Using the previously discussed closure properties we can derive a useful characterization of the Borel classes which we formulate in the following proposition.

Proposition 3.12 (Characterization of multiplicative sets) Let $X$ be a computable metric space. Then the function

$$
Z: \boldsymbol{\Sigma}_{k}^{0}(X \rightarrow[0,1]) \rightarrow \boldsymbol{\Pi}_{k}^{0}(X), f \mapsto f^{-1}\{0\}
$$

is computable and it admits a computable right inverse.

Proof. Since $\{0\} \in \boldsymbol{\Pi}_{1}^{0}([0,1])$, it follows directly that $f^{-1}\{0\} \in \boldsymbol{\Pi}_{k}^{0}(X)$ for every function $f \in \Sigma_{k}^{0}(X \rightarrow[0,1])$ and $Z$ is even computable. Now we prove that $Z$ admits a computable right inverse $Z^{-}: \boldsymbol{\Pi}_{k}^{0}(X) \rightrightarrows \boldsymbol{\Sigma}_{k}^{0}(X \rightarrow[0,1])$. In case $k=1$ this is well-known (see Theorem 3.10 in [7]). Thus, let us consider the case $k>1$. To this end, let $A \in \boldsymbol{\Pi}_{k}^{0}(X)$. Then we can effectively find a sequence $\left(U_{i}\right)_{i \in \mathbb{N}}$ in $\Sigma_{k-1}^{0}(X)$ such that $A=\bigcap_{i=0}^{\infty} U_{i}$. Now consider the characteristic functions

$$
\operatorname{cf}_{U_{i}}: X \rightarrow[0,1], x \mapsto\left\{\begin{array}{ll}
0 & \text { if } x \in U_{i} \\
1 & \text { else }
\end{array} .\right.
$$

It is easy to see that the sequence $\left(\mathrm{cf}_{U_{i}}\right)_{i \in \mathbb{N}}$ is a computable sequence in $\boldsymbol{\Sigma}_{k}^{0}(X \rightarrow[0,1])$. We define $f: X \rightarrow[0,1]$ by $f(x):=\sum_{i=0}^{\infty} \operatorname{cf}_{U_{i}}(x) 2^{-i-1}$ for all $x \in X$. Then we can effectively find $f \in \Sigma_{k}^{0}(X \rightarrow[0,1])$ by Proposition 3.10 and $f(x)=0 \Longleftrightarrow(\forall i) \operatorname{cf}_{U_{i}}(x)=0 \Longleftrightarrow x \in A$.

This enables us to prove a generalization of the (weak) Graph Theorem for computable functions. 
Theorem 3.13 (Graph Theorem) Let $X$ and $Y$ be computable metric spaces and let $k \geq 1$. The mapping

$$
\operatorname{graph}: \boldsymbol{\Sigma}_{k}^{0}(X \rightarrow Y) \rightarrow \boldsymbol{\Pi}_{k}^{0}(X \times Y), f \mapsto \operatorname{graph}(f)
$$

is computable.

Proof. We consider the computable metric spaces $X$ and $(Y, d, \alpha)$. Since $d: Y \times Y \rightarrow \mathbb{R}$ is computable, we obtain that $U: X \times Y \rightarrow[0,1],(x, y) \mapsto \max (d(f(x), y), 1)$ is $\boldsymbol{\Sigma}_{k}^{0}$-measurable for all $\boldsymbol{\Sigma}_{k}^{0}$-measurable $f: X \rightarrow Y$ and

$$
U(x, y)=0 \Longleftrightarrow d(f(x), y)=0 \Longleftrightarrow f(x)=y \Longleftrightarrow(x, y) \in \operatorname{graph}(f) .
$$

Thus $U^{-1}\{0\}=\operatorname{graph}(f)$ and by Proposition 3.12 and Proposition 3.8 it follows that $\operatorname{graph}(f)=$ $Z\left(\max \circ\left(d \circ\left(f \times \operatorname{id}_{Y}\right), 1\right)\right)$ is computable (where $1: X \times Y \rightarrow \mathbb{R}$ denotes the constant function with value 1$)$.

The partial inverse of the mapping graph is not computable in general. Even in case $k=1$ this does only hold for certain $X, Y$ (e.g. if $Y$ is recursive compact, see [6] for a detailed discussion of this problem).

\section{Effective selection theorems}

In this section we study some effective versions of selection theorems. This section contains the technical core of this paper and the reader who would like to follow the main results might skip this section during the first reading.

One message of this section could be reduced to the slogan that "single-valuedness is for free in case of higher order Borel computability". More precisely: in case of $k \geq 2$ any $\boldsymbol{\Sigma}_{k}^{0}$ computable multi-valued operation $F: X \rightrightarrows Y$ with closed images has a $\boldsymbol{\Sigma}_{k}^{0}$-computable singlevalued selector (provided that $Y$ is complete). This is in harsh contrast to the situation of $k=1$ where it is known that many natural problems (like determination of zeros) admit computable multi-valued solutions but no single-valued ones. We will establish this result by proving an effective version of the Kuratowski-Ryll-Nardzewski Selection Theorem [14].

In a second step we will even prove an (essentially) stronger selection theorem which allows to conclude that (under certain assumptions) the composition $F \circ \Delta$ of two $\boldsymbol{\Sigma}_{2}^{0}$-computable mappings mappings $\Delta: X \rightrightarrows W$ and $F: W \rightarrow Z$ has a single-valued $\boldsymbol{\Sigma}_{2}^{0}$-computable selector $s: X \rightarrow Z$. This is not obvious at all since in a general context at most $\boldsymbol{\Sigma}_{3}^{0}$-computability could be concluded. A first such section theorem has been established by Saint Raymond [19] and it has been generalized to a selection theorem by Bhattacharya-Srivastava [1]. We will prove an effective version of this Selection Theorem which is our main tool in order to obtain transfer results in later sections. The classical version of the Bhattacharya-Srivastava Selection Theorem not only generalizes the Section Theorem of Saint Raymond but it also implies the Selection Theorem of Kuratowski and Ryll-Nardzewski. Although our proof follows very closely the original proof given by Bhattacharya and Srivastava, the effectivization needs some care. Especially, we have to restrict the theorem to metric spaces with recursive open balls, a property which we will define below and which has not been required classically. As a consequence, the effective version of the Bhattacharya-Srivastava Selection Theorem does (formally) not imply the effective version of the Kuratowski-Ryll-Nardzewski Selection Theorem which we will present 
now. While some of the forementioned classical selection theorems are presented in the very general context of set fields, we will formulate all our results in the context of additive Borel classes. The proof of the following result is a direct translation of the constructive classical proof. By $\bar{A}$ we denote the topological closure of the set $A$.

Theorem 4.1 (Effective Kuratowski-Ryll-Nardzewski Selection Theorem) Let $X, Y$ be computable metric spaces and let $Y$ be complete and $k \geq 2$. There is a computable operation $S: \Sigma_{k}^{0}(X \rightrightarrows Y) \rightrightarrows \Sigma_{k}^{0}(X \rightarrow Y)$ such that $f(x) \in \overline{F(x)}$ for any $f \in S(F), F \in \Sigma_{k}^{0}(X \rightrightarrows Y)$ and $x \in X$.

Proof. We consider the computable metric spaces $X$ and $(Y, d, \alpha)$. Without loss of generality we can assume that the diameter of $Y$ is less than 1 (otherwise we use the computationally equivalent metric $d^{\prime}(x, y):=d(x, y) /(1+2 d(x, y))$, see [3]). Given a $\boldsymbol{\Sigma}_{k}^{0}$-measurable operation $F: X \rightrightarrows Y$ we will define a sequence of $\Sigma_{k}^{0}$-measurable mappings $f_{n}: X \rightarrow Y$ which fulfill

$$
\begin{array}{r}
d_{F(x)}\left(f_{n}(x)\right)<2^{-n}, \\
d\left(f_{n}(x), f_{n-1}(x)\right)<2^{-n}
\end{array}
$$

for any $n \in \mathbb{N}$ and we will apply Proposition 3.10 to this sequence. (Here and in the following $d_{A}: Y \rightarrow \mathbb{R}, y \mapsto \inf _{a \in A} d(a, y)$ denotes the distance function of $A \subseteq Y$.) We will define the sequence $\left(f_{n}\right)_{n \in \mathbb{N}}$ inductively. Let $f_{0}(x):=\alpha(0)$ for all $x \in X$. Then $f_{0}$ is obviously $\boldsymbol{\Sigma}_{k}^{0}$ computable and fulfills Equation (1) and (2) (if we set $f_{-1}:=f_{0}$ ). Now let us assume that for some $n>0$ the function $f_{n-1}$ is $\boldsymbol{\Sigma}_{k}^{0}$-measurable and fulfills Equations (1) and (2). We define for all $i \in \mathbb{N}$

- $C_{i}:=\left\{x \in X: d_{F(x)}(\alpha(i))<2^{-n}\right\}$,

- $D_{i}:=\left\{x \in X: d\left(\alpha(i), f_{n-1}(x)\right)<2^{-n+1}\right\}$,

- $A_{i}:=C_{i} \cap D_{i}$.

Then we obtain $X=\bigcup_{i=0}^{\infty} A_{i}$ since for any $x \in X$ there is by Equation (1) a point $y \in F(x)$ such that $d\left(y, f_{n-1}(x)\right)<2^{-n+1}$ and there is some $i \in \mathbb{N}$ such that $d(\alpha(i), y)<2^{-n}$ and additionally $d(\alpha(i), y)+d\left(y, f_{n-1}(x)\right)<2^{-n+1}$. Hence $x \in A_{i}$. On the other hand, we obtain

- $C_{i}=\left\{x \in X: F(x) \cap B\left(\alpha(i), 2^{-n}\right) \neq \emptyset\right\}=F^{-1}\left(B\left(\alpha(i), 2^{-n}\right)\right)$,

- $D_{i}=f_{n-1}^{-1}\left(B\left(\alpha(i), 2^{-n+1}\right)\right)$.

Thus, by assumption we can effectively compute $C_{i}, D_{i} \in \boldsymbol{\Sigma}_{k}^{0}(X)$ and hence $A_{i} \in \boldsymbol{\Sigma}_{k}^{0}(X)$, effectively in $i$ (since intersection is computable by Proposition 3.2). Since $k \geq 2$, we can find a sequence of sets $E_{\langle i, j\rangle} \in \Pi_{k-1}^{0}(X)$ such that $A_{i}=\bigcup_{j=0}^{\infty} E_{\langle i, j\rangle}$ for all $i$ and thus $X=\bigcup_{s=0}^{\infty} E_{s}$. Let $k_{s}, m_{s} \in \mathbb{N}$ be such that $\left\langle k_{s}, m_{s}\right\rangle=s$ for all $s \in \mathbb{N}$. Now we define $f_{n}: X \rightarrow Y$ for any $s \in \mathbb{N}$ by $f_{n}(x)=\alpha\left(k_{s}\right)$ for all $x \in E_{s} \backslash\left(E_{0} \cup \ldots \cup E_{s-1}\right)$. By definition we obtain

$$
f_{n}^{-1}\{\alpha(i)\}=\bigcup\left\{E_{s} \backslash\left(E_{0} \cup \ldots \cup E_{s-1}\right): s \in \mathbb{N} \text { and } k_{s}=i\right\} \in \boldsymbol{\Sigma}_{k}^{0}(X)
$$

and by Proposition 3.2 we can effectively determine these sets. Similarly, we can determine

$$
f_{n}^{-1}\left(B\left(\alpha(j), 2^{-m}\right)\right)=\bigcup\left\{f^{-1}\{\alpha(i)\}: i \in \mathbb{N} \text { and } d(\alpha(i), \alpha(j))<2^{-m}\right\} \in \boldsymbol{\Sigma}_{k}^{0}(X)
$$


for all $j, m \in \mathbb{N}$ and thus $f_{n}$ is $\boldsymbol{\Sigma}_{k}^{0}$-measurable. We still have to prove that $f_{n}$ satisfies Equations (1) and (2). Therefore, let $x \in X$ and let $s \in \mathbb{N}$ be such that $x \in E_{s} \backslash\left(E_{0} \cup \ldots \cup E_{s-1}\right)$. With $i:=k_{s}$ we obtain $f_{n}(x)=\alpha(i)$ and $x \in E_{s} \subseteq A_{i}=C_{i} \cap D_{i}$. Now $x \in C_{i}$ implies Equation (1) and $x \in D_{i}$ implies Equation (2). By Equation (2) and since $Y$ is complete it follows that $\left(f_{n}\right)_{n \in \mathbb{N}}$ converges pointwise to a function $f: X \rightarrow Y$ which is $\boldsymbol{\Sigma}_{k}^{0}$-measurable and can be effectively determined by Proposition 3.10. By Equation (1) it follows that $f(x) \in \overline{F(x)}$ for any $x \in X$.

We immediately obtain the following non-uniform version of this result which in particular states that any $\boldsymbol{\Sigma}_{k}^{0}$-computable operation with closed images admits an $\boldsymbol{\Sigma}_{k}^{0}$-computable selector (for any $k \geq 2$ ). This indicates that for applications multi-valued operations are less important in higher Borel classes.

Corollary 4.2 Let $X, Y$ are computable metric spaces and let $Y$ be complete and $k \geq 2$. Then for any $\boldsymbol{\Sigma}_{k}^{0}$-computable operation $F: X \rightrightarrows Y$ there is a $\boldsymbol{\Sigma}_{k}^{0}$-computable function $f: X \rightarrow Y$ with $f(x) \in \overline{F(x)}$ for all $x \in X$.

Especially, this implies that any computable operation admits a $\boldsymbol{\Sigma}_{2}^{0}$-computable selector. This is due to the fact that computable operations admit computable set-valued selectors with compact images (this can be deduced, for instance, from Lemma 6.3).

Corollary 4.3 Let $X, Y$ be computable metric spaces and let $Y$ be complete. Any computable operation $F: \subseteq X \rightrightarrows Y$ admits a $\boldsymbol{\Sigma}_{2}^{0}$-computable selector $f: \subseteq X \rightarrow Y$ with $f(x) \in F(x)$ for all $x \in X$.

It is known that in case of $Y=\mathbb{N}^{\mathbb{N}}$ or $Y=\{0,1\}^{\mathbb{N}}$ the selector can even be computable itself (see Theorem 3.2.11 in [3]). The previous corollaries have the following direct consequence (where $\mathcal{K}(X)$ denotes the computable metric space of non-empty compact subsets, endowed with the Hausdorff metric; similar results can be obtained for closed subsets).

Corollary 4.4 (Choice) For any complete computable metric space $X$ there is a $\boldsymbol{\Sigma}_{2}^{0}$-computable choice function choice $: \mathcal{K}(X) \rightarrow X$ with choice $(A) \in A$ for any $A \in \mathcal{K}(X)$.

This follows from the fact that the multi-valued choice map Choice : $\mathcal{K}(X) \rightrightarrows X$ with $\operatorname{Choice}(A)=A$ is computable.

Now we continue with an effective version of the Bhattacharya-Srivastava Selection Theorem [1]. The forementioned authors have called a multi-valued operation $F: X \rightrightarrows Y$ strongly $\boldsymbol{\Sigma}_{k}^{0}$-measurable, if $F^{-1}(A) \in \boldsymbol{\Sigma}_{k}^{0}(X)$ for any closed set $A \subseteq Y$. Following this terminology we introduce strong effective $\Sigma_{k}^{0}$-measurability.

Definition 4.5 (Strong Borel computability) Let $X, Y$ be computable metric spaces. A multi-valued operation $F: \subseteq X \rightrightarrows Y$ is called strongly effectively $\boldsymbol{\Sigma}_{k}^{0}-$ measurable or strongly $\boldsymbol{\Sigma}_{k}^{0}-$ computable, if there exists a computable operation $\Phi: \boldsymbol{\Pi}_{1}^{0}(Y) \rightrightarrows \boldsymbol{\Sigma}_{k}^{0}(X)$ such that $F^{-1}(A)=$ $B \cap \operatorname{dom}(F)$ for any $A \in \Pi_{1}^{0}(Y)$ and $B \in \Phi(A)$.

Now we introduce computable metric spaces with recursive open balls, another property which appears as a premise in the effective version of the Bhattacharya-Srivastava Selection Theorem below. 
Definition 4.6 (Recursive open balls) A computable metric space $(X, d, \alpha)$ is said to have recursive open balls, if $(B(\alpha(i), \bar{j}))_{\langle i, j\rangle \in \mathbb{N}}$ is a computable sequence of recursive open sets (or equivalently, if $\left(d_{X \backslash B(\alpha(i), \bar{j})}^{-1}(-\infty, \bar{r})\right)_{\langle i, j, r\rangle \in \mathbb{N}}$ is a computable sequence in $\left.\boldsymbol{\Sigma}_{1}^{0}(X)\right)$.

Many computable metric spaces (such as $\mathbb{R}, \mathbb{N}^{\mathbb{N}}$ etc.) have recursive open balls. However, it is not too difficult to construct computable metric spaces which do not have recursive balls. Now we are prepared to formulate and prove an effective version of the Selection Theorem of Bhattacharya and Srivastava. We do not claim that the property of recursive open balls is necessary for the following result (it might even be superfluous). However, it is sufficient to effectivize the original proof. To make the statement more readable we formulate it informally. By $\mathbb{N}^{*}$ we denote the set of finite words over $\mathbb{N}$, by $\varepsilon \in \mathbb{N}^{*}$ the empty word, by $\lg (w)$ the length of the word $w \in \mathbb{N}^{*}$ and by $p[i]=p(0) \ldots p(i-1) \in \mathbb{N}^{*}$ the prefix of $p \in \mathbb{N}^{\mathbb{N}}$ of length $i \in \mathbb{N}$.

Theorem 4.7 (Effective Bhattacharya-Srivastava Selection Theorem) Let $X, W, Z$ be computable metric spaces, let $W$ be complete with recursive open balls and let $k \geq 2$. For any closed valued strongly $\boldsymbol{\Sigma}_{k}^{0}-$ measurable $\Delta: X \rightrightarrows W$ and any given $\boldsymbol{\Sigma}_{2}^{0}$-measurable function $F: \subseteq W \rightarrow Z$ with range $(\Delta) \subseteq \operatorname{dom}(F)$ we can effectively find a $\boldsymbol{\Sigma}_{k}^{0}$-measurable function $s: X \rightarrow W$ such that $s(x) \in \Delta(x)$ for any $x \in X$ and $F \circ s: X \rightarrow Z$ is $\boldsymbol{\Sigma}_{k}^{0}$-measurable.

Proof. We consider the computable metric spaces $(W, d, \alpha),\left(Z, d^{\prime}, \beta\right)$ and $\left(X, d^{\prime \prime}, \gamma\right)$ and $k \geq 2$. Without loss of generality, we can assume that the diameters of $W$ and $Z$ are less than 1 (otherwise we use the computationally equivalent metric $(x, y) \mapsto d(x, y) /(1+2 d(x, y))$ in case of $W$ and correspondingly in case of $Z$, see [3]). Given a strongly $\boldsymbol{\Sigma}_{k}^{0}$-measurable operation $\Delta: X \rightrightarrows W$ with closed values and a $\Sigma_{2}^{0}$-measurable function $F: \subseteq W \rightarrow Z$ we will define subsets $X_{w} \subseteq X$ and $W_{w} \subseteq W$ for all finite words $w \in \mathbb{N}^{*}$ such that

(1) $X_{w} \in \Sigma_{k}^{0}(X), X_{\varepsilon}=X$,

(2) $X_{w n} \cap X_{w m}=\emptyset$ for all $n \neq m$,

(3) $X_{w}=\bigcup_{j=0}^{\infty} X_{w j}$,

(4) $W_{w} \in \Pi_{1}^{0}(W)$ and $\operatorname{diam}\left(W_{w}\right)<2^{-l}$,

(5) $\operatorname{diam}\left(F\left(W_{w}\right)\right)<2^{-l}$,

(6) $\Delta(x) \cap W_{w} \neq \emptyset$ for any $x \in X_{w}$

holds for all $w \in \mathbb{N}^{l}$ and $l \in \mathbb{N}$. Inductively, we start with $X_{\varepsilon}:=X$ and $W_{\varepsilon}:=W$. Now let $l \in \mathbb{N}$ and let us assume that $X_{w}$ and $W_{w}$ have been defined according to (1)-(6) for all $w \in \bigcup_{i=0}^{l} \mathbb{N}^{i}$. Let us fix such a $w$ an let $A_{\left\langle m_{1}, m_{2}\right\rangle}:=\bar{B}\left(\alpha\left(m_{1}\right), 2^{-m_{2}-l-2}\right) \subseteq W$ and $V_{\left\langle i_{1}, i_{2}\right\rangle}:=$ $B\left(\beta\left(i_{1}\right), 2^{-i_{2}-l-2}\right) \subseteq Z$. By assumption on $F$ we can effectively find sets $E_{\langle i, j\rangle} \in \Pi_{1}^{0}(W)$ such that

$$
F^{-1}\left(V_{i}\right)=\operatorname{dom}(F) \cap \bigcup_{j=0}^{\infty} E_{\langle i, j\rangle}
$$

for any $i \in \mathbb{N}$. Now let for all $n, m \in \mathbb{N}$

$$
P_{w,\langle n, m\rangle}:=\left\{x \in X_{w}: \Delta(x) \cap W_{w} \cap A_{m} \cap E_{n} \neq \emptyset\right\} \subseteq X .
$$


Since $A_{\left\langle m_{1}, m_{2}\right\rangle}=W \backslash \bigcup\left\{B(\alpha(i), \bar{j}): d\left(\alpha(i), \alpha\left(m_{1}\right)\right)>2^{-m_{2}-l-1}+\bar{j}\right\}$, for any $m=\left\langle m_{1}, m_{2}\right\rangle \in \mathbb{N}$, we can determine $A_{m} \in \Pi_{1}^{0}(W)$ and hence by Proposition 3.2 we can effectively determine $W_{w} \cap A_{m} \cap E_{n} \in \Pi_{1}^{0}(W)$ for any $n, m \in \mathbb{N}$. Since $\Delta$ is strongly $\boldsymbol{\Sigma}_{k}^{0}$-measurable, it follows that we can effectively determine $P_{w,\langle n, m\rangle}=\Delta^{-1}\left(W_{w} \cap A_{m} \cap E_{n}\right) \in \Sigma_{k}^{0}(X)$ and since $k \geq 2$ we can find $Q_{w,\langle\langle n, m\rangle, i\rangle} \in \Pi_{k-1}^{0}(X)$ such that $P_{w,\langle n, m\rangle}=\bigcup_{i=0}^{\infty} Q_{w,\langle\langle n, m\rangle, i\rangle}$. Consequently, we can effectively determine

$$
X_{w 0}:=Q_{w, 0} \in \boldsymbol{\Sigma}_{k}^{0}(X) \text { and } X_{w n}:=Q_{w, n} \backslash\left(\bigcup_{i=0}^{n-1} Q_{w, i}\right) \in \boldsymbol{\Sigma}_{k}^{0}(X)
$$

for all $n \geq 1$ by Proposition 3.2. We obtain $\bigcup_{n=0}^{\infty} X_{w n}=\bigcup_{n=0}^{\infty} P_{w, n}=X_{w}$ since range $(\Delta) \subseteq$ $\operatorname{dom}(F)$ and by definition $X_{w n} \cap X_{w m}=\emptyset$ whenever $n \neq m$. Finally, we can effectively determine

$$
W_{w\langle\langle n, m\rangle, i\rangle}:=W_{w} \cap A_{m} \cap E_{n} \in \mathbf{\Pi}_{1}^{0}(W)
$$

for any $n, m, i \in \mathbb{N}$. We still have to prove that $X_{w n}$ and $W_{w\langle\langle n, m\rangle, i\rangle}$ fulfill (1)-(6) for any $n, m, i \in \mathbb{N}$. We have already seen that (1)-(3) hold. Moreover, (4) holds since $W_{w\langle\langle n, m\rangle, i\rangle} \subseteq A_{m}$ and $\operatorname{diam}\left(A_{m}\right)<2^{-l}$. Similarly (5) holds, since $W_{w\langle\langle n, m\rangle, i\rangle} \subseteq E_{n}, F\left(E_{\left\langle n_{1}, n_{2}\right\rangle}\right) \subseteq V_{n_{1}}$ and $\operatorname{diam}\left(V_{n_{1}}\right)<2^{-l}$ for $n=\left\langle n_{1}, n_{2}\right\rangle \in \mathbb{N}$ (here we assume $\operatorname{diam}(\emptyset)=0$ ). Finally, (6) holds since $x \in X_{w\langle\langle n, m\rangle, i\rangle}$ implies $x \in Q_{w,\langle\langle n, m\rangle, i\rangle} \subseteq P_{w,\langle n, m\rangle}$ and hence $\Delta(x) \cap W_{w\langle\langle n, m\rangle, i\rangle} \neq \emptyset$.

Now we will use the sets with properties (1)-(6) in order to construct a selector $s: X \rightarrow W$ of $\Delta$. For any $x \in X$ there is a unique $p \in \mathbb{N}^{\mathbb{N}}$ such that $x \in X_{p[i]}$ for all $i \in \mathbb{N}$ by (1)-(3). By (4) and (6) and since $W$ is complete and $\Delta$ has closed images it follows that $\bigcap_{i=0}^{\infty} W_{p[i]} \subseteq \Delta(x)$ is a singleton. Let $s(x)$ be defined by $\{s(x)\}=\bigcap_{i=0}^{\infty} W_{p[i]}$. Thus, it is clear that $s$ is a selector of $\Delta$, i.e. $s(x) \in \Delta(x)$. In order to prove that $s$ is $\Sigma_{k}^{0}$-measurable we define $\Delta_{l}: X \rightrightarrows W$ by

$$
\Delta_{l}(x):=\Delta(x) \cap W_{p[l]} \text { if } x \in X_{p[l]}
$$

for any $x \in X, p \in \mathbb{N}^{\mathbb{N}}$ and $l \in \mathbb{N}$. For any $A \in \Pi_{1}^{0}(W)$ we obtain

$$
\Delta_{l}^{-1}(A)=\bigcup_{w \in \mathbb{N}^{l}}\left\{x \in X_{w}: \Delta(x) \cap W_{w} \cap A \neq \emptyset\right\}=\bigcup_{w \in \mathbb{N}^{l}}\left(X_{w} \cap \Delta^{-1}\left(W_{w} \cap A\right)\right)
$$

and thus $\Delta_{l}$ is strongly $\boldsymbol{\Sigma}_{k}^{0}$-measurable since $\Delta$ is. Since $X$ has recursive open balls, we can effectively determine

$$
A_{l}:=\left\{y \in W: B\left(y, 2^{-l}\right) \subseteq B\left(\alpha\left(j_{1}\right), \overline{j_{2}}\right)\right\}=W \backslash\left\{y \in W: d_{W \backslash B\left(\alpha\left(j_{1}\right), \overline{j_{2}}\right)}(y)<2^{-l}\right\} \in \mathbf{\Pi}_{1}^{0}(W)
$$

for all $j_{1}, j_{2}, l \in \mathbb{N}$. Now using (3) and (4) we obtain

$$
\begin{aligned}
s^{-1}\left(B\left(\alpha\left(j_{1}\right), \overline{j_{2}}\right)\right) & =\bigcup_{l=0}^{\infty}\left\{x \in X: B\left(s(x), 2^{-l}\right) \subseteq B\left(\alpha\left(j_{1}\right), \overline{j_{2}}\right)\right\} \\
& =\bigcup_{l=0}^{\infty} \bigcup_{w \in \mathbb{N}^{l}}\left\{x \in X_{w}:\left(\exists y \in \Delta(x) \cap W_{w}\right) B\left(y, 2^{-l}\right) \subseteq B\left(\alpha\left(j_{1}\right), \overline{j_{2}}\right)\right\} \\
& =\bigcup_{l=0}^{\infty}\left\{x \in X: \Delta_{l}(x) \cap A_{l} \neq \emptyset\right\} \\
& =\bigcup_{l=0}^{\infty} \Delta_{l}^{-1}\left(A_{l}\right)
\end{aligned}
$$


and thus $s$ is $\boldsymbol{\Sigma}_{k}^{0}$-measurable. We still have to prove that $F \circ s: X \rightarrow Z$ is $\boldsymbol{\Sigma}_{k}^{0}$-measurable. To this end, we first prove that $F \circ \Delta_{l}: X \rightrightarrows Z$ is $\boldsymbol{\Sigma}_{k}^{0}$-measurable. This follows, since for any $l, i \in \mathbb{N}$

$$
\begin{aligned}
\left(F \circ \Delta_{l}\right)^{-1}\left(V_{i}\right) & =\Delta_{l}^{-1}\left(\bigcup_{j=0}^{\infty} E_{\langle i, j\rangle}\right) \\
& =\bigcup_{j=0}^{\infty}\left\{x \in X: \Delta_{l}(x) \cap E_{\langle i, j\rangle} \neq \emptyset\right\} \\
& =\bigcup_{j=0}^{\infty} \bigcup_{w \in \mathbb{N}^{l}}\left\{x \in X_{w}: \Delta(x) \cap W_{w} \cap E_{\langle i, j\rangle} \neq \emptyset\right\} \\
& =\bigcup_{j=0}^{\infty} \bigcup_{w \in \mathbb{N}^{l}}\left(X_{w} \cap \Delta^{-1}\left(W_{w} \cap E_{\langle i, j\rangle}\right)\right) \in \Sigma_{k}^{0}(X) .
\end{aligned}
$$

Altogether we have seen that we can effectively determine the sequence $\left(F \circ \Delta_{l}\right)_{l \in \mathbb{N}}$ of operations $F \circ \Delta_{l}: X \rightrightarrows Z$ in $\Sigma_{k}^{0}(X \rightrightarrows Z)$. By (5), (6) it follows that this sequence fulfills the premises of Proposition 3.10, thus $F \circ s=\operatorname{Lim}\left(F \circ \Delta_{l+1}\right)_{l \in \mathbb{N}} \in \Sigma_{k}^{0}(X \rightarrow Z)$ can be effectively determined.

A precise version of the formulation of this theorem would be the following: Let $X, W, Z$ be computable metric spaces, let $W$ be complete with recursive open balls and let $k \geq 2$. For any closed valued strongly $\Sigma_{k}^{0}$-measurable $\Delta: X \rightrightarrows W$ with $D:=\operatorname{dom}(\Delta)$ there is a computable operation $S:\left.\boldsymbol{\Sigma}_{2}^{0}(W \rightarrow Z)\right|_{D} \rightrightarrows \boldsymbol{\Sigma}_{k}^{0}(X \rightarrow W) \times \boldsymbol{\Sigma}_{k}^{0}(X \rightarrow Z)$ such that $s(x) \in \Delta(x)$ for any $x \in X$ and $G=F \circ s$ for all $(s, G) \in S(F)$. However, this formulation is not uniform in $\Delta$ while the proof is. Thus, we could formulate yet another uniform version using some representation $\delta$ of the set of strongly $\Sigma_{k}^{0}$-measurable functions $\Delta: X \rightrightarrows W$ which could be defined analogously to $\delta_{\boldsymbol{\Sigma}_{k}^{0}(X \rightrightarrows W)}$. We leave these extentions to the reader.

\section{Reducibility of functions}

In this section we will study reducibility of functions and we will show that on Baire space there are complete functions in the classes of Borel measurable functions. Reducibility of functions has been defined and studied by Weihrauch and others [26, 24, 25, 22, 16, 9].

Definition 5.1 (Reducibility) Let $X, Y, U, V$ be computable metric spaces and consider functions $f: \subseteq X \rightarrow Y$ and $g: \subseteq U \rightarrow V$. We say that $f$ is reducible to $g$, for short $f \leqslant_{\mathrm{t}} g$, if there are continuous functions $A: \subseteq X \times V \rightarrow Y$ and $B: \subseteq X \rightarrow U$ such that

$$
f(x)=A(x, g \circ B(x))
$$

for all $x \in \operatorname{dom}(f)$. Correspondingly, $f$ is called computably reducible to $g$, for short $f \leqslant_{\mathrm{c}} g$, if there are corresponding $A, B$ which are computable. The corresponding equivalences are denoted by $\cong_{\mathrm{t}}$ and $\cong_{\mathrm{c}}$.

The reader should notice that we have used slightly other symbols for the reducibilities and equivalences in order to distinguish them from the corresponding relations for representations. It is easy to see that the relations $\leqslant_{\mathrm{t}}$ and $\leqslant_{\mathrm{c}}$ are reflexive and transitive (this is due to the fact 
that computable and continuous operations are closed under composition). The formula of the reducibility can also be written as $f(x)=A \circ(\mathrm{id}, g \circ B)(x)$ and using it we can conclude the following result which states that (computable) reducibility preserves (effective) measurability.

Proposition 5.2 Let $X, Y, U$ and $V$ be computable metric spaces and let us consider two partial functions $f: \subseteq X \rightarrow Y$ and $g: \subseteq U \rightarrow V$. Then the following holds for all $k \geq 1$ :

(1) $f \leqslant_{\mathrm{t}} g$ and $g$ is $\boldsymbol{\Sigma}_{k}^{0}$-measurable $\Longrightarrow f$ is $\boldsymbol{\Sigma}_{k}^{0}$-measurable,

(2) $f \leqslant_{c} g$ and $g$ is $\boldsymbol{\Sigma}_{k}^{0}$-computable $\Longrightarrow f$ is $\boldsymbol{\Sigma}_{k}^{0}$-computable.

The proof directly follows from the closure properties of $\boldsymbol{\Sigma}_{k}^{0}$-measurable functions in Proposition 3.8. Now we are prepared to define functions which will turn out to be complete with respect to reducibility in certain classes of Borel measurable functions on Baire space. The definition is motivated by the logical Tarski-Kuratowski characterization of Borel classes [15]. We use a standard tupling $\left\langle p, n_{1}, \ldots, n_{k}\right\rangle \in \mathbb{N}^{\mathbb{N}}$ for $p \in \mathbb{N}^{\mathbb{N}}$ and $n_{1}, \ldots, n_{k} \in \mathbb{N}$. It is known that such a tupling function can be chosen such that it is computable, bijective and the inverse is computable as well.

Definition 5.3 For any $k \in \mathbb{N}$ we define $C_{k}: \mathbb{N}^{\mathbb{N}} \rightarrow \mathbb{N}^{\mathbb{N}}$ by

$$
C_{k}(p)(n):= \begin{cases}0 & \text { if }\left(\exists n_{k}\right)\left(\forall n_{k-1}\right) \ldots p\left\langle n, n_{1}, \ldots, n_{k}\right\rangle \neq 0 \\ 1 & \text { otherwise }\end{cases}
$$

for all $p \in \mathbb{N}^{\mathbb{N}}$ and $n \in \mathbb{N}$.

Here (and similarly in the following) we assume that the quantifiers $\forall$ and $\exists$ are alternating and the final quantification is $\left(\forall n_{1}\right)$ in case that $k$ is even and $\left(\exists n_{1}\right)$ in case that $k$ is odd. It should be noticed that $C_{0}$ is a computable function and it is easy to see that the mapping $C_{1}$ is computationally equivalent to the map

$$
C(p)(n):= \begin{cases}0 & \text { if }(\exists m) p(m)=n+1 \\ 1 & \text { otherwise }\end{cases}
$$

which translates enumerations of sets into their characteristic functions. We prove that $C_{k}$ is complete in the class of all $\Sigma_{k}^{0}$-computable functions on Baire space. The proof is based on the following normal form result.

Lemma 5.4 (Effective Tarski-Kuratowski normal form) For any $k \geq 1$ the map

$$
L_{k}: \boldsymbol{\Delta}_{1}^{0}\left(\mathbb{N}^{\mathbb{N}}\right) \rightarrow \Sigma_{k}^{0}\left(\mathbb{N}^{\mathbb{N}}\right), A \mapsto\left\{p \in \mathbb{N}^{\mathbb{N}}:\left(\exists n_{k}\right)\left(\forall n_{k-1}\right) \ldots\left\langle p, n_{1}, n_{2}, \ldots, n_{k}\right\rangle \in A\right\}
$$

is computable and it admits a computable multi-valued right inverse.

Proof. We consider the computable metric space $\left(\mathbb{N}^{\mathbb{N}}, d, \alpha\right)$. Since the injections $\boldsymbol{\Delta}_{1}^{0} \hookrightarrow \boldsymbol{\Sigma}_{1}^{0}$ and $\boldsymbol{\Delta}_{1}^{0} \hookrightarrow \boldsymbol{\Pi}_{1}^{0}$ are computable by definition, it follows by applying the closure schemes from Proposition 3.2 that $L_{k}$ is computable. It remains to prove that $L_{k}$ admits a computable right inverse $L_{k}^{-}: \boldsymbol{\Sigma}_{k}^{0}\left(\mathbb{N}^{\mathbb{N}}\right) \rightrightarrows \boldsymbol{\Delta}_{1}^{0}\left(\mathbb{N}^{\mathbb{N}}\right)$. We will prove this by induction on $k$. In case $k=1$ we can effectively find $B\left(\alpha\left(m_{i}\right), \overline{r_{i}}\right)$ for any given $U \in \boldsymbol{\Sigma}_{1}^{0}\left(\mathbb{N}^{\mathbb{N}}\right)$ such that $U=\bigcup_{i=0}^{\infty} B\left(\alpha\left(m_{i}\right), \overline{r_{i}}\right)$. 
Let $B:=\bigcup_{i=0}^{\infty}\left(B\left(\alpha\left(m_{i}\right), \overline{r_{i}}\right) \times\left\{\left\langle m_{i}, r_{i}\right\rangle\right\}\right)$. In the special case of Baire space $\mathbb{N}^{\mathbb{N}}$, all balls $B\left(\alpha\left(m_{i}\right), \overline{r_{i}}\right)$ are open as well as closed and by Lemma 3.3 we can even effectively determine $B\left(\alpha\left(m_{i}\right), \overline{r_{i}}\right) \in \mathbf{\Pi}_{1}^{0}\left(\mathbb{N}^{\mathbb{N}}\right)$. Thus, we can also determine

$$
\left(B\left(\alpha\left(m_{i}\right), \overline{r_{i}}\right) \times\left\{\left\langle m_{i}, r_{i}\right\rangle\right\}\right)^{c}=\left(B\left(\alpha\left(m_{i}\right), \overline{r_{i}}\right)^{c} \times\left\{\left\langle m_{i}, r_{i}\right\rangle\right\}\right) \cup\left(B\left(\alpha\left(m_{i}\right), \overline{r_{i}}\right) \times\left(\mathbb{N} \backslash\left\{\left\langle m_{i}, r_{i}\right\rangle\right\}\right)\right)
$$

in $\Pi_{1}^{0}\left(\mathbb{N}^{\mathbb{N}} \times \mathbb{N}\right)$ and consequently $B \in \boldsymbol{\Delta}_{1}^{0}\left(\mathbb{N}^{\mathbb{N}} \times \mathbb{N}\right)$. Since the tupling function \langle\rangle$: \mathbb{N}^{\mathbb{N}} \times \mathbb{N} \rightarrow \mathbb{N}^{\mathbb{N}}$ and its inverse are computable, we can effectively find

$$
A:=\langle B\rangle=\left\{\langle p, n\rangle \in \mathbb{N}^{\mathbb{N}}:(p, n) \in B\right\} \in \Delta_{1}^{0}\left(\mathbb{N}^{\mathbb{N}}\right)
$$

and we obtain $L_{1}(A)=U$. This finishes the proof in case of $k=1$. Now let us assume that we have proved the statement for some $k \geq 1$ and let us conclude that it holds for $k+1$ as well. If $U \in \boldsymbol{\Sigma}_{k+1}^{0}\left(\mathbb{N}^{\mathbb{N}}\right)$, then there are $V_{i} \in \Pi_{k}^{0}\left(\mathbb{N}^{\mathbb{N}}\right)$ such that $U=\bigcup_{i=0}^{\infty} V_{i}$. For any $i$ we can find a set $A_{i} \in \boldsymbol{\Delta}_{1}^{0}\left(\mathbb{N}^{\mathbb{N}}\right)$ such that $L_{k}\left(A_{i}\right)=V_{i}^{c}$ by induction hypothesis. Similar as in the case $k=1$ we can effectively determine $B:=\bigcup_{i=0}^{\infty}\left(A_{i}^{c} \times\{i\}\right) \in \boldsymbol{\Delta}_{1}^{0}\left(\mathbb{N}^{\mathbb{N}} \times \mathbb{N}\right)$ and $A:=\left\{\left\langle p, n_{1}, \ldots, n_{k+1}\right\rangle \in \mathbb{N}^{\mathbb{N}}:\left(\left\langle p, n_{1}, \ldots, n_{k}\right\rangle, n_{k+1}\right) \in B\right\} \in \boldsymbol{\Delta}_{1}^{0}\left(\mathbb{N}^{\mathbb{N}}\right)$. It follows

$$
\begin{aligned}
L_{k+1}(A) & =\left\{p \in \mathbb{N}^{\mathbb{N}}:\left(\exists n_{k+1}\right)\left(\forall n_{k}\right) \ldots\left(\left\langle p, n_{1}, n_{2}, \ldots, n_{k}\right\rangle, n_{k+1}\right) \in B\right\} \\
& =\left\{p \in \mathbb{N}^{\mathbb{N}}:(\exists i)\left(\forall n_{k}\right) \ldots\left\langle p, n_{1}, n_{2}, \ldots, n_{k}\right\rangle \in A_{i}^{c}\right\} \\
& =\left\{p \in \mathbb{N}^{\mathbb{N}}:(\exists i) p \in V_{i}\right\} \\
& =U .
\end{aligned}
$$

Using this logical normal form we can now prove the following theorem.

Theorem 5.5 (Completeness) Let $k \in \mathbb{N}$. For any function $F: \subseteq \mathbb{N}^{\mathbb{N}} \rightarrow \mathbb{N}^{\mathbb{N}}$ we obtain:

(1) $F \leqslant_{\mathrm{t}} C_{k} \Longleftrightarrow F$ is $\Sigma_{k+1}^{0}$-measurable,

(2) $F \leqslant_{\mathrm{c}} C_{k} \Longleftrightarrow F$ is $\Sigma_{k+1}^{0}-$ computable.

Proof. For the "only if" direction of the proofs it suffices by Proposition 5.2 to show that $C_{k}$ is $\boldsymbol{\Sigma}_{k+1}^{0}$-computable. Using Lemma 5.4 it follows that

$$
R_{n}:=\left\{p \in \mathbb{N}^{\mathbb{N}}:\left(\exists n_{k}\right)\left(\forall n_{k-1}\right) \ldots p\left\langle n, n_{1}, \ldots, n_{k}\right\rangle \neq 0\right\}
$$

defines a computable sequence $\left(R_{n}\right)_{n \in \mathbb{N}}$ in $\boldsymbol{\Sigma}_{k}^{0}\left(\mathbb{N}^{\mathbb{N}}\right)$. Since

$$
C_{k}^{-1}\left(a_{0} \ldots a_{i} \mathbb{N}^{\mathbb{N}}\right)=\bigcup_{\substack{n=0 \\ a_{n}=0}}^{i} R_{n} \cap \bigcup_{\substack{n=0 \\ a_{n}=1}}^{i} R_{n}^{\mathrm{c}}
$$

it follows that $C_{k}$ is $\boldsymbol{\Sigma}_{k+1}^{0}$-computable.

It remains to prove the "if" direction. We consider the computable case (2). To this end we first prove that it suffices to consider functions $F: \subseteq \mathbb{N}^{\mathbb{N}} \rightarrow \mathbb{N}^{\mathbb{N}}$ with range $(F) \subseteq\{0,1\}^{\mathbb{N}}$. This is due to the fact that the canonical injection $\iota: \mathbb{N}^{\mathbb{N}} \rightarrow \mathbb{N}^{\mathbb{N}}, p \mapsto 0^{p(0)+1} 10^{p(1)+1} 10^{p(2)+1} 1 \ldots$ and its 
partial inverse $\iota^{-1}$ are computable and if we can prove $\iota \circ F \leqslant_{\mathrm{c}} C_{k}$, then $F \leqslant_{\mathrm{c}} \iota F \leqslant_{\mathrm{c}} C_{k}$ follows. Thus, let us assume without loss of generality that $F: \subseteq \mathbb{N}^{\mathbb{N}} \rightarrow \mathbb{N}^{\mathbb{N}}$ is $\boldsymbol{\Sigma}_{k+1}^{0}$-computable and range $(F) \subseteq\{0,1\}^{\mathbb{N}}$. Using the effective Tarski-Kuratowski normal form from Lemma 5.4, we can conclude that we can find computable sets $P, Q \in \Delta_{1}^{0}\left(\mathbb{N}^{\mathbb{N}}\right)$ such that

$$
F(p)(n)= \begin{cases}0 & \text { if }\left(\exists n_{k+1}\right)\left(\forall n_{k}\right) \ldots\left\langle p, n, n_{1}, \ldots, n_{k+1}\right\rangle \in P \\ 1 & \text { if }\left(\exists n_{k+1}\right)\left(\forall n_{k}\right) \ldots\left\langle p, n, n_{1}, \ldots, n_{k+1}\right\rangle \in Q\end{cases}
$$

for all $p \in \operatorname{dom}(F)$ and $n \in \mathbb{N}$. We first claim $F \leqslant{ }_{\mathrm{c}} C_{k} \times C_{k}$. We define $A: \subseteq \mathbb{N}^{\mathbb{N}} \times \mathbb{N}^{\mathbb{N}} \rightarrow \mathbb{N}^{\mathbb{N}}$ by

$$
A(p, q)(n)= \begin{cases}0 & \text { if }(\exists m) p\langle n, m\rangle=1 \\ 1 & \text { if }(\exists m) q\langle n, m\rangle=1\end{cases}
$$

with $\operatorname{dom}(A)=\{(p, q):(\forall n)(((\exists m)(p\langle n, m\rangle=1)) \oplus((\exists m)(q\langle n, m\rangle=1)))\}$, where " $\oplus$ " denotes the exclusive or. Moreover, let $B_{P}: \mathbb{N}^{\mathbb{N}} \rightarrow \mathbb{N}^{\mathbb{N}}$ be defined by

$$
B_{P}(p)\left\langle\langle n, m\rangle, n_{1}, \ldots, n_{k}\right\rangle:= \begin{cases}0 & \left\langle p, n, n_{1}, \ldots, n_{k}, m\right\rangle \in P \\ 1 & \text { otherwise }\end{cases}
$$

and define $B_{Q}: \mathbb{N}^{\mathbb{N}} \rightarrow \mathbb{N}^{\mathbb{N}}$ analogously. Let $B:=\left(B_{P}, B_{Q}\right): \mathbb{N}^{\mathbb{N}} \rightarrow \mathbb{N}^{\mathbb{N}} \times \mathbb{N}^{\mathbb{N}}$. By definition $A, B$ are computable and we obtain for all $p \in \operatorname{dom}(F)$ and $n, m \in \mathbb{N}$

$$
\begin{aligned}
C_{k} \circ B_{P}(p)\langle n, m\rangle=1 & \Longleftrightarrow\left(\forall n_{k}\right)\left(\exists n_{k-1}\right) \ldots B_{P}(p)\left\langle\langle n, m\rangle, n_{1}, \ldots, n_{k}\right\rangle=0 \\
& \Longleftrightarrow\left(\forall n_{k}\right)\left(\exists n_{k-1}\right) \ldots\left\langle p, n, n_{1}, \ldots, n_{k}, m\right\rangle \in P
\end{aligned}
$$

and analogously with $Q$ instead of $P$. In particular, we obtain $\left(C_{k} \times C_{k}\right) \circ B(p) \in \operatorname{dom}(A)$ for all $p \in \operatorname{dom}(F)$ and thus

$$
\begin{aligned}
A \circ\left(C_{k} \times C_{k}\right) \circ B(p)(n)=0 & \Longleftrightarrow(\exists m) C_{k} \circ B_{P}(p)\langle n, m\rangle=1 \\
& \Longleftrightarrow(\exists m)\left(\forall n_{k}\right)\left(\exists n_{k-1}\right) \ldots\left\langle p, n, n_{1}, \ldots, n_{k}, m\right\rangle \in P \\
& \Longleftrightarrow F(p)(n)=0
\end{aligned}
$$

for all $p \in \operatorname{dom}(F)$ and $n \in \mathbb{N}$. Hence, $F \leqslant{ }_{c} C_{k} \times C_{k}$. It remains to prove $C_{k} \times C_{k} \leqslant{ }_{\mathrm{c}} C_{k}$. To this end we define $D: \mathbb{N}^{\mathbb{N}} \rightarrow \mathbb{N}^{\mathbb{N}} \times \mathbb{N}^{\mathbb{N}}$ by $D\langle p, q\rangle:=(p, q)$ and $E: \mathbb{N}^{\mathbb{N}} \times \mathbb{N}^{\mathbb{N}} \rightarrow \mathbb{N}^{\mathbb{N}}$ by

$$
E(p, q)\left\langle m, n_{1}, \ldots, n_{k}\right\rangle:= \begin{cases}p\left\langle n, n_{1}, \ldots, n_{k}\right\rangle & \text { if } m=2 n \\ q\left\langle n, n_{1}, \ldots, n_{k}\right\rangle & \text { if } m=2 n+1\end{cases}
$$

for all $p, q \in \mathbb{N}^{\mathbb{N}}$ and $n, m, n_{1}, \ldots, n_{k} \in \mathbb{N}$. Then $D, E$ are computable and we obtain

$$
C_{k}(p)(n)=0 \Longleftrightarrow C_{k} \circ E(p, q)(2 n)=0 \text { and } C_{k}(q)(n)=0 \Longleftrightarrow C_{k} \circ E(p, q)(2 n+1)=0
$$

and thus $C_{k} \times C_{k}(p, q)=D \circ C_{k} \circ E(p, q)$, i.e. $C_{k} \times C_{k} \leqslant c C_{k}$. Here we assume that $\langle p, q\rangle(2 n)=p(n)$ and $\langle p, q\rangle(2 n+1)=q(n)$.

The proof for the continuous case (1) is completely analogous.

A careful inspection of the proof shows that we have not employed the full complexity of the reducibility. More precisely, let us define $\Gamma: \subseteq \mathbb{N}^{\mathbb{N}} \rightarrow \mathbb{N}^{\mathbb{N}}$ by $\Gamma:=\iota^{-1} A D$ with the notations from the proof. Then $\Gamma$ is computable and does not depend on $F$ and we obtain the following result. 
Corollary 5.6 Let $k \geq 1$ and let $F: \subseteq \mathbb{N}^{\mathbb{N}} \rightarrow \mathbb{N}^{\mathbb{N}}$ be a function. Then $F \leqslant_{\mathrm{t}} C_{k}$ holds, if and only if there is a continuous function $B: \subseteq \mathbb{N}^{\mathbb{N}} \rightarrow \mathbb{N}^{\mathbb{N}}$ such that $F(p)=\Gamma \circ C_{k} \circ B(p)$ for all $p \in \operatorname{dom}(F)$. An analogous statement holds with respect to $\leqslant_{\mathrm{c}}$ with computable $B$.

This allows to define the following representation of $\boldsymbol{\Sigma}_{k}^{0}\left(\mathbb{N}^{\mathbb{N}} \rightarrow \mathbb{N}^{\mathbb{N}}\right)$.

Definition 5.7 For any $k \in \mathbb{N}$ and any computable metric subspace $D \subseteq \mathbb{N}^{\mathbb{N}}$ we define a representation $\delta_{C_{k}}$ of $\boldsymbol{\Sigma}_{k}^{0}\left(D \rightarrow \mathbb{N}^{\mathbb{N}}\right)$ by

$$
\delta_{C_{k}}(p):=\Gamma \circ C_{k} \circ \delta_{\boldsymbol{\Sigma}_{1}^{0}\left(D \rightarrow \mathbb{N}^{\mathbb{N}}\right)}(q)
$$

for all $p \in \mathbb{N}^{\mathbb{N}}$ and $f \in \Sigma_{k}^{0}\left(D \rightarrow \mathbb{N}^{\mathbb{N}}\right)$.

Since the proof of the Completeness Theorem is fully effective, we can formulate the following effective version (for the case $D=\mathbb{N}^{\mathbb{N}}$ ).

Corollary 5.8 (Effective Completeness Theorem) $\delta_{C_{k}} \equiv_{c} \delta_{\Sigma_{k+1}^{0}\left(\mathbb{N}^{\mathbb{N}} \rightarrow \mathbb{N}^{\mathbb{N}}\right)}$ for any $k \in \mathbb{N}$.

As we have seen, the degrees of non-computability are additive with respect to composition. We will close this section by proving that any $\boldsymbol{\Sigma}_{k}^{0}$-computable function can be decomposed in a composition of $\boldsymbol{\Sigma}_{1}^{0}$-computable functions.

Lemma 5.9 (Factorization) The function $F_{k}: \mathbb{N}^{\mathbb{N}} \rightarrow \mathbb{N}^{\mathbb{N}}$, defined by

$$
F_{k}(p)\langle n, m\rangle:= \begin{cases}1 & \text { if }\left(\forall n_{k}\right)\left(\exists n_{k-1}\right) \ldots p\left\langle n, n_{1}, \ldots, n_{k}, m\right\rangle \neq 0 \\ 0 & \text { otherwise }\end{cases}
$$

for all $p \in \mathbb{N}^{\mathbb{N}}$ and $n, m \in \mathbb{N}$, is $\boldsymbol{\Sigma}_{k+1}^{0}$-computable for any $k \in \mathbb{N}$ and $C_{k+1}=C_{1} \circ F_{k}$.

In particular, this results shows that a function $F: \subseteq \mathbb{N}^{\mathbb{N}} \rightarrow \mathbb{N}^{\mathbb{N}}$ is $C^{k}$-continuous (in the sense studied in $[22,16])$, if and only if $F$ is $\boldsymbol{\Sigma}_{k+1}^{0}-$ measurable.

\section{The Representation Theorem}

In this section we will extend the Kreitz-Weihrauch Representation Theorem [13] (see also $[23,26,21,5])$ to Borel measurable mappings on computable metric spaces. The classical theorem states that a total function $f: X \rightarrow Y$ (on separable metric spaces $X, Y$ and even on larger classes of spaces) is continuous, if and only if it admits a continuous realizer $F$, see Figure 1. Moreover, computable functions are defined via computable realizers. We will prove that this result extends to Borel measurable functions on all finite levels. It turns out that the effective Bhattacharya-Srivastava Selection Theorem 4.7 can be employed for this purpose. Before we start with the technical part of this section, we just formulate our main result.

Theorem 6.1 (Representation Theorem) Let $X, Y$ be computable metric spaces, $k \geq 1$ and let $f: X \rightarrow Y$ be a total function. Then $f$ is (effectively) $\Sigma_{k}^{0}$-measurable, if and only if $f$ admits an (effectively) $\Sigma_{k}^{0}$-measurable realizer $F: \subseteq \mathbb{N}^{\mathbb{N}} \rightarrow \mathbb{N}^{\mathbb{N}}$.

For the proof of this result we will apply the effective Bhattacharya-Srivastava Selection Theorem combined with certain representations introduced by Schröder [20], which we will define next. 
Definition 6.2 Let $(X, d, \alpha)$ be a separable metric space. Define $\sigma_{X}: \subseteq \mathbb{N}^{\mathbb{N}} \rightarrow X$ by

$$
\sigma_{X}(p)=x: \Longleftrightarrow p \in\{0,1\}^{\mathbb{N}} \text { and }(\forall i, j \in \mathbb{N})\left\{\begin{array}{l}
p\langle i, j\rangle=0 \Longrightarrow d(x, \alpha(i))<2^{-j} \\
p\langle i, j\rangle=1 \Longrightarrow d(x, \alpha(i))>2^{-j-1}
\end{array}\right.
$$

for all $x \in X$ and $p \in \mathbb{N}$. Similarly, we define $\widehat{\sigma}_{X}: \subseteq \mathbb{N}^{\mathbb{N}} \rightarrow X$ with " $<$ " and "> " replaced by " $\leq$ " and " $\geq$ ", respectively.

One important property of these representations is that they have compact fibers (and $\widehat{\sigma}_{X}$ is even proper, i.e. preimages of compact sets are compact). We will in particular employ those properties of Schröder's representation which are given in the following lemma. Some of these properties have essentially been proved in [27] and [20]. We recall that the hyperspace $\mathcal{K}(X)$ of compact subsets of $X$ can be endowed with different representations [7, 27]. Here we just mention that we denote by $\mathcal{K}_{<}(X)$ this space endowed with the "positive representation" (a name of a set $A$ is a list of all open rational balls which intersect $A$ ) and by $\mathcal{K}_{>}(X)$ we denote the space endowed with the "negative representation" (a name of a set $A$ is a list of all finite covers of $A$ by open rational balls).

Lemma 6.3 Let $X$ be a computable metric space. Then the following holds:

(1) $\sigma_{X} \equiv_{\mathrm{c}} \widehat{\sigma}_{X} \equiv_{\mathrm{c}} \delta_{X}$ (computably admissible).

(2) $\widehat{\kappa}_{X}: \mathcal{K}_{>}(X) \rightarrow \mathcal{K}_{>}\left(\mathbb{N}^{\mathbb{N}}\right), K \mapsto \widehat{\sigma}_{X}^{-1}(K)$ is computable (computably proper).

(3) $\Phi_{X}: \Gamma\left(\mathbb{N}^{\mathbb{N}}\right) \rightarrow \Gamma(X), A \mapsto \widehat{\sigma}_{X}(A)$ is computable for $\Gamma \in\left\{\boldsymbol{\Pi}_{1}^{0}, \boldsymbol{\Sigma}_{2}^{0}\right\}$.

(4) $\widehat{\Delta}_{X}: X \rightrightarrows \mathbb{N}^{\mathbb{N}}, x \mapsto \widehat{\sigma}_{X}^{-1}\{x\}$ is strongly $\Sigma_{2}^{0}$-computable.

(5) $\kappa_{X}: X \rightarrow \mathcal{K}_{<}\left(\mathbb{N}^{\mathbb{N}}\right), x \mapsto \sigma_{X}^{-1}\{x\}$ is computable (computably fiber compact).

(6) $\Delta_{X}: X \rightrightarrows \mathbb{N}^{\mathbb{N}}, x \mapsto \sigma_{X}^{-1}\{x\}$ is $\Sigma_{1}^{0}$-computable.

Proof. (1) and (2) have been proved in [27] and [20]. It remains to prove (3)-(6). Now for any $A \in \Pi_{1}^{0}(X)$ we obtain

$$
\Phi_{X}(A)=\left\{x \in X: \widehat{\sigma}_{X}^{-1}\{x\} \cap A \neq \emptyset\right\}=X \backslash\left\{x \in X: \widehat{\kappa}_{X}(\{x\}) \subseteq\left(\mathbb{N}^{\mathbb{N}} \backslash A\right)\right\} \in \mathbf{\Pi}_{1}^{0}(X)
$$

by (2). Thus $\Phi_{X}$ is computable for $\Gamma=\boldsymbol{\Pi}_{1}^{0}$. It directly follows that $\Phi_{X}$ is computable for $\Gamma=\boldsymbol{\Sigma}_{2}^{0}$ since $\Phi_{X}\left(\bigcup_{i=0}^{\infty} A_{i}\right)=\bigcup_{i=0}^{\infty} \Phi_{X}\left(A_{i}\right)$. Since $\widehat{\Delta}_{X}^{-1}(A)=\Phi_{X}(A)$ and $\boldsymbol{\Pi}_{1}^{0}(X) \hookrightarrow \boldsymbol{\Sigma}_{2}^{0}(X)$ is computable, we obtain (4) from (3) case $\Gamma=\Pi_{1}^{0}$. Since

$$
\Delta_{X}^{-1}(B(y, r))=\left\{x \in X: \kappa_{X}(x) \cap B(y, r) \neq \emptyset\right\} \in \Sigma_{1}^{0}(X),
$$

we obtain (6) from (5). It remains to prove (5). For the proof we consider the computable metric space $(X, d, \alpha)$. One verifies that $\sigma_{X}(p) \in \sigma_{X}\left(w \mathbb{N}^{\mathbb{N}}\right)$, if for all $\langle i, j\rangle \leq \lg (w)-1$ there exists $\left\langle i^{\prime}, j^{\prime}\right\rangle$ such that $p\left\langle i^{\prime}, j^{\prime}\right\rangle=0$ and

$$
\left\{\begin{array}{l}
w(\langle i, j\rangle)=0 \Longrightarrow d\left(\alpha(i), \alpha\left(i^{\prime}\right)\right)<2^{-j}-2^{-j^{\prime}} \\
w(\langle i, j\rangle)=1 \Longrightarrow d\left(\alpha(i), \alpha\left(i^{\prime}\right)\right)>2^{-j-1}+2^{-j^{\prime}}
\end{array}\right.
$$


for all $p \in \operatorname{dom}\left(\sigma_{X}\right)$ and $w \in \mathbb{N}^{*}$. Since $X$ is a computable metric space, it follows that $M=\left\{(p, w) \in \mathbb{N}^{\mathbb{N}} \times \mathbb{N}^{*}: \sigma_{X}(p) \in \sigma_{X}\left(w \mathbb{N}^{\mathbb{N}}\right)\right\}$ is r.e. in $\operatorname{dom}\left(\sigma_{X}\right) \times \mathbb{N}^{*}$. This, implies that given $p \in \operatorname{dom}\left(\sigma_{X}\right)$, we can effectively enumerate the set $\left\{w \in \mathbb{N}^{*}: w \mathbb{N}^{\mathbb{N}} \cap \sigma_{X}^{-1} \sigma_{X}(p) \neq \emptyset\right\}$ which implies the claim since $\operatorname{dom}\left(\sigma_{X}\right) \subseteq\{0,1\}^{\mathbb{N}}$.

Now we prove a section theorem which can be considered as an effective version of the Section Theorem of Saint Raymond [19], generalized to arbitrary separable metric spaces, but specialized to Cauchy representations.

Proposition 6.4 (Section) Let $X$ be a computable metric space and let $D:=\operatorname{dom}\left(\delta_{X}\right)$. Then there is a computable operation $S: \boldsymbol{\Sigma}_{k}^{0}\left(D \rightarrow \mathbb{N}^{\mathbb{N}}\right) \rightrightarrows \boldsymbol{\Sigma}_{2}^{0}\left(X \rightarrow \mathbb{N}^{\mathbb{N}}\right) \times \boldsymbol{\Sigma}_{k}^{0}\left(X \rightarrow \mathbb{N}^{\mathbb{N}}\right)$ for any $k \geq 2$ such that $\delta_{X} \circ s(x)=x$ and $g=F \circ s$ for all $\Sigma_{k}^{0}$-measurable functions $F: D \rightarrow \mathbb{N}^{\mathbb{N}}$ and $(s, g) \in S(F)$.

Proof. We will prove the claim by induction on $k \geq 2$. By Lemma 6.3 we have $\widehat{\sigma}_{X} \leq_{\mathrm{c}} \delta_{X}$ and thus there is some computable function $G: \subseteq \mathbb{N}^{\mathbb{N}} \rightarrow \mathbb{N}^{\mathbb{N}}$ such that $\widehat{\sigma}_{X}(p)=\delta_{X} G(p)$ for all $p \in$ $\operatorname{dom}\left(\widehat{\sigma}_{X}\right)$. Given $F \in \boldsymbol{\Sigma}_{k}^{0}\left(D \rightarrow \mathbb{N}^{\mathbb{N}}\right)$ we can effectively determine $\left.F \circ G \in \boldsymbol{\Sigma}_{k}^{0}\left(\mathbb{N}^{\mathbb{N}} \rightarrow \mathbb{N}^{\mathbb{N}}\right)\right|_{\operatorname{dom}\left(\widehat{\sigma}_{X}\right)}$. By Lemma 6.3 we know that $\widehat{\Delta}_{X}: X \rightrightarrows \mathbb{N}^{\mathbb{N}}$ is strongly $\Sigma_{2}^{0}$-computable. Moreover, $\mathbb{N}^{\mathbb{N}}$ is complete and has recursive open balls. Thus, the statement for $k=2$ is a direct consequence of the effective Bhattacharya-Srivastava Selection Theorem 4.7, applied to $\widehat{\Delta}_{X}$ and $F G$. It states that we can effectively find a $\boldsymbol{\Sigma}_{2}^{0}$-measurable selector $t: X \rightarrow \mathbb{N}^{\mathbb{N}}$ of $\widehat{\Delta}_{X}$ and that we can effectively determine $g:=F G \circ t \in \boldsymbol{\Sigma}_{k}^{0}\left(X \rightarrow \mathbb{N}^{\mathbb{N}}\right)$. Thus we can also effectively find $s:=G \circ t \in \Sigma_{2}^{0}\left(X \rightarrow \mathbb{N}^{\mathbb{N}}\right)$ and we obtain

$$
\delta_{X} \circ s(x)=\delta_{X} \circ G \circ t(x)=\widehat{\sigma}_{X} \circ t(x)=x
$$

and $g(x)=F \circ G \circ t(x)=F \circ s(x)$ for all $x \in X$. The lower part of the diagram in Figure 2 illustrates the situation.

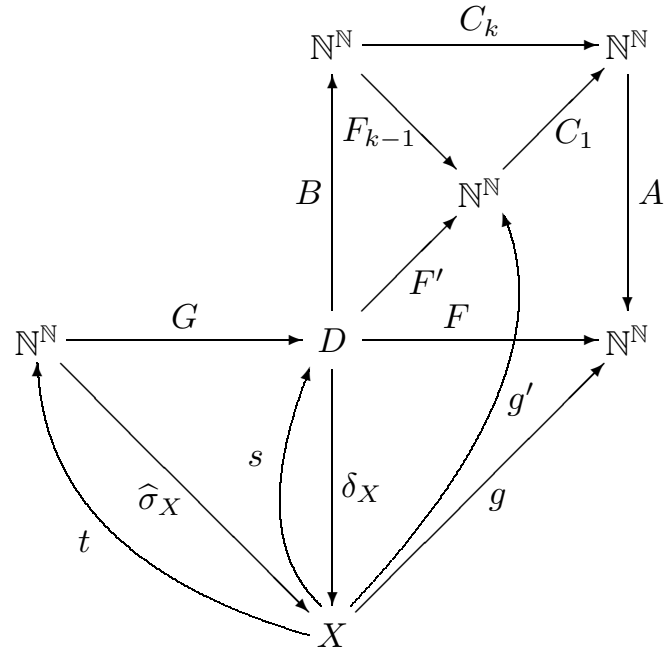

Figure 2: Diagram for the proof

Let us assume that we have already proved the theorem for some $k \geq 2$. We will show that it holds for $k+1$. Given $F \in \Sigma_{k+1}^{0}\left(D \rightarrow \mathbb{N}^{\mathbb{N}}\right)$, we can effectively find some continuous functions 
$A: \subseteq \mathbb{N}^{\mathbb{N}} \rightarrow \mathbb{N}^{\mathbb{N}}$ and $B: D \rightarrow \mathbb{N}^{\mathbb{N}}$ such that $F(p)=A \circ C_{k} \circ B(p)$ for all $p \in \operatorname{dom}(F)=D$ by the effective Completeness Theorem, Corollary 5.8. By Lemma 5.9 we can effectively find a $\Sigma_{k}^{0}$-computable function $F_{k-1}$ such that $C_{k}=C_{1} \circ F_{k-1}$. Thus, we can effectively determine $F^{\prime}:=F_{k-1} \circ B \in \boldsymbol{\Sigma}_{k}^{0}\left(D \rightarrow \mathbb{N}^{\mathbb{N}}\right)$. We apply the induction hypothesis to $F^{\prime}$ and we obtain functions $s \in \Sigma_{2}^{0}\left(X \rightarrow \mathbb{N}^{\mathbb{N}}\right)$ and $g^{\prime} \in \Sigma_{k}^{0}\left(X \rightarrow \mathbb{N}^{\mathbb{N}}\right)$ such that $\delta_{X} \circ s(x)=x$ and $g^{\prime}=F^{\prime} \circ s$. Hence

$$
g:=F s=A C_{k} B s=A C_{1} F_{k-1} B s=A C_{1} F^{\prime} s=A C_{1} g^{\prime} \in \Sigma_{k+1}^{0}\left(X \rightarrow \mathbb{N}^{\mathbb{N}}\right) .
$$

The right part of the diagram in Figure 2 illustrates the situation. This finishes the induction.

Now we can easily derive a proof of the Representation Theorem 6.1.

Proof. (Representation Theorem 6.1) The result for $k=1$ is well-known and thus we can assume $k \geq 2$. Let $\delta_{X}$ and $\delta_{Y}$ be the Cauchy representations of $X$ and $Y$, respectively with $D:=\operatorname{dom}\left(\delta_{X}\right)$ and let $\Delta_{Y}: Y \rightrightarrows \mathbb{N}^{\mathbb{N}}$ be defined as in Lemma 6.3.

Let $f$ admit a realizer $F \in \boldsymbol{\Sigma}_{k}^{0}\left(D \rightarrow \mathbb{N}^{\mathbb{N}}\right)$. Then by Proposition 6.4 we can effectively determine a selector $s: X \rightarrow \mathbb{N}^{\mathbb{N}}$ of $\delta_{X}$ such that $F \circ s \in \boldsymbol{\Sigma}_{k}^{0}\left(X \rightarrow \mathbb{N}^{\mathbb{N}}\right)$. Hence we can effectively determine $f=\delta_{Y} \circ F \circ s \in \boldsymbol{\Sigma}_{k}^{0}(X \rightarrow Y)$.

Now let on the other hand $f \in \boldsymbol{\Sigma}_{k}^{0}(X \rightarrow Y)$. By Lemma 6.3 $\Delta_{Y}: Y \rightrightarrows \mathbb{N}^{\mathbb{N}}$ is $\boldsymbol{\Sigma}_{1}^{0}$-computable and thus we can determine $\Delta_{Y} \circ f \circ \delta_{X} \in \Sigma_{k}^{0}\left(D \rightrightarrows \mathbb{N}^{\mathbb{N}}\right)$. Moreover, $\Delta_{Y}$ has closed images and hence by the effective Kuratowski-Ryll-Nardzewski Selection Theorem 4.1 we can find a selector $t \in \boldsymbol{\Sigma}_{k}^{0}\left(D \rightarrow \mathbb{N}^{\mathbb{N}}\right)$ of $\Delta_{Y} \circ f \circ \delta_{X}$. Since $\sigma_{Y} \leq_{\mathrm{c}} \delta_{Y}$ by Lemma 6.3 , there is a computable $G: \subseteq \mathbb{N}^{\mathbb{N}} \rightarrow \mathbb{N}^{\mathbb{N}}$ such that $\sigma_{Y}(p)=\delta_{Y} G(p)$ for all $p \in \operatorname{dom}\left(\sigma_{Y}\right)=\operatorname{range}\left(\Delta_{Y}\right)$. Thus, we can effectively determine $F=G \circ t \in \boldsymbol{\Sigma}_{k}^{0}\left(D \rightarrow \mathbb{N}^{\mathbb{N}}\right)$ and $\delta_{Y} F=\delta_{Y} G t=\sigma_{Y} t=f \delta_{X}$, i.e. $F$ is a $\Sigma_{k}^{0}$-measurable realizer of $f$.

Although the theorem has not been formulated uniformly, the proof is completely uniform. Before we formulate this uniform version, we mention that the previous theorem allows to define the following realizability representation of Borel measurable functions.

Definition 6.5 Let $\delta_{X}$ and $\delta_{Y}$ be Cauchy representations of metric spaces $X$ and $Y$ respectively, let $D:=\operatorname{dom}\left(\delta_{X}\right)$ and let $k \geq 1$. Define a representation $\boldsymbol{\Sigma}_{k}^{0}\left[\delta_{X} \rightarrow \delta_{Y}\right]$ of the $\boldsymbol{\Sigma}_{k}^{0}$-measurable functions $f: X \rightarrow Y$ by

$$
\Sigma_{k}^{0}\left[\delta_{X} \rightarrow \delta_{Y}\right](p)=f: \Longleftrightarrow(\forall q \in D) f \circ \delta_{X}(q)=\delta_{Y} \circ\left(\delta_{\boldsymbol{\Sigma}_{k}^{0}\left(D \rightarrow \mathbb{N}^{\mathbb{N}}\right)}(p)\right)(q)
$$

for all $p \in \mathbb{N}^{\mathbb{N}}$ and $f \in \boldsymbol{\Sigma}_{k}^{0}(X \rightarrow Y)$.

Using this representation, we can formulate the effective version of the Representation Theorem.

Corollary 6.6 (Effective Representation Theorem) Let $X$ and $Y$ be computable metric space with Cauchy representations $\delta_{X}$ and $\delta_{Y}$, respectively. Then $\boldsymbol{\Sigma}_{k}^{0}\left[\delta_{X} \rightarrow \delta_{Y}\right] \equiv_{c_{c}} \delta_{\boldsymbol{\Sigma}_{k}^{0}(X \rightarrow Y)}$ for any $k \geq 1$.

The reader should notice that the presented material allows an elementary proof of the Representation Theorem (i.e. a proof without application of any selection theorems) at least 
in case $k \leq 2$. On the one hand, one can use the Tarski-Kuratowski normal form of the preimages $\left(f \delta_{X}\right)^{-1}(U) \subseteq \mathbb{N}^{\mathbb{N}}$ in order to prove that any $\boldsymbol{\Sigma}_{k}^{0}$-computable function $f$ admits a $\boldsymbol{\Sigma}_{k}^{0}$-computable realizer (this even works for all $k \geq 1$ ). On the other hand, Lemma 6.3 (3) for $\Gamma=\boldsymbol{\Sigma}_{2}^{0}$ implies that $f=\delta_{Y} F \widehat{\Delta}_{X}$ is $\boldsymbol{\Sigma}_{2}^{0}$-computable whenever $f$ admits a $\boldsymbol{\Sigma}_{2}^{0}$-computable realizer $F$ (with respect to $\widehat{\sigma}_{X}, \delta_{X}$ ). We close this section with the following Transfer Theorem which allows to conclude computability properties of multiplicative sets from their name sets.

Theorem 6.7 (Transfer Theorem) Let $X$ be a computable metric space with Cauchy representation $\delta_{X}$. Then the map $T: \subseteq \Pi_{k}^{0}\left(\mathbb{N}^{\mathbb{N}}\right) \rightarrow \Pi_{k}^{0}(X), \delta_{X}^{-1}(A) \mapsto A$ is computable for any $k \geq 1$.

Proof. We first consider the case $k \geq 2$. Let $A \subseteq X$. If $\delta_{X}^{-1}(A) \in \Pi_{k}^{0}\left(\mathbb{N}^{\mathbb{N}}\right)$ is given, then by Proposition 3.12 we can effectively find a function $H \in \Sigma_{k}^{0}\left(\mathbb{N}^{\mathbb{N}} \rightarrow[0,1]\right)$ such that $H^{-1}\{0\}=$ $\delta_{X}^{-1}(A)$. By the Representation Theorem 6.1 there is some realizer $F \in \Sigma_{k}^{0}\left(\mathbb{N}^{\mathbb{N}} \rightarrow \mathbb{N}^{\mathbb{N}}\right)$ of $H$, i.e. $\delta_{[0,1]} \circ F=H$. By Proposition 6.4 there is a function $s: X \rightarrow \mathbb{N}^{\mathbb{N}}$ with $\delta_{X} \circ s(x)=x$ such that we can effectively determine $F \circ s \in \boldsymbol{\Sigma}_{k}^{0}\left(X \rightarrow \mathbb{N}^{\mathbb{N}}\right)$ and we obtain $f:=\delta_{[0,1]} \circ F \circ s \in \boldsymbol{\Sigma}_{k}^{0}(X \rightarrow[0,1])$ and $f^{-1}\{0\}=\left(\delta_{[0,1]} \circ F \circ s\right)^{-1}\{0\}=s^{-1} H^{-1}\{0\}=s^{-1}\left(\delta_{X}^{-1}(A)\right)=A$. Hence, by Proposition 3.12 we obtain $A \in \Pi_{k}^{0}(X)$.

In case $k=1$ we consider the representation $\sigma_{X}$. Since $\sigma_{X} \equiv_{\mathrm{c}} \delta_{X}$, there is a computable function $G: \subseteq \mathbb{N}^{\mathbb{N}} \rightarrow \mathbb{N}^{\mathbb{N}}$ such that $\sigma_{X}(p)=\delta_{X} G(p)$ for all $p \in \operatorname{dom}\left(\sigma_{X}\right)$. If $\delta_{X}^{-1}(A) \in \Pi_{1}^{0}\left(\mathbb{N}^{\mathbb{N}}\right)$ is given, then we can effectively determine a set $B \in \Pi_{1}^{0}\left(\mathbb{N}^{\mathbb{N}}\right)$ such that $G^{-1} \delta_{X}^{-1}(A)=B \cap \operatorname{dom}\left(\sigma_{X}\right)$. Hence, by Proposition 3.12 , we can effectively find a function $F \in \Sigma_{1}^{0}\left(\mathbb{N}^{\mathbb{N}} \rightarrow[0,1]\right)$ such that $F^{-1}\{0\}=B$. Then by Lemma $6.3 \Delta_{X}: X \rightrightarrows \mathbb{N}^{\mathbb{N}}$ is $\Sigma_{1}^{0}$-computable and hence $F \Delta_{X}$ is $\boldsymbol{\Sigma}_{1}^{0}$-computable. Since $[0,1]$ is complete and $\Delta_{X}$ and hence $F \Delta_{X}$ have compact images, we can effectively find a selector $s \in \boldsymbol{\Sigma}_{1}^{0}(X \rightarrow[0,1])$ of $F \Delta_{X}$ (see the remark after Corollary 4.3). Now $s(x)=0$ implies that there is some $p \in \Delta_{X}(x) \cap B$ and this in turn implies $x=\sigma_{X}(p)=\delta_{X} G(p) \in A$. On the other hand, $x \in A$ implies $G^{-1} \delta_{X}^{-1}\{x\} \subseteq B \cap \operatorname{dom}\left(\sigma_{X}\right)$ and hence $s(x) \in F \circ \Delta_{X}(x)=\{0\}$, i.e. $s(x)=0$. Altogether, $s^{-1}\{0\}=A$ and thus $A \in \mathbf{\Pi}_{1}^{0}(X)$ by Proposition 3.12 .

Since $\mathbb{N}^{\mathbb{N}} \backslash \delta_{X}^{-1}(A)=D^{c} \cup \delta_{X}^{-1}(X \backslash A)$ and $D:=\operatorname{dom}\left(\delta_{X}\right) \in \Pi_{2}^{0}\left(\mathbb{N}^{\mathbb{N}}\right)$ in case that $X$ is complete, we can conclude an analog statement for additive sets at least in case $k>1$. Altogether, we obtain the following corollary.

Corollary 6.8 Let $X$ be a (complete) computable metric space with Cauchy representation $\delta_{X}$. Let $A \subseteq X$ and let $k \geq 1$. If $\delta_{X}^{-1}(A)$ is a computable $\boldsymbol{\Pi}_{k}^{0}$-set (or a computable $\boldsymbol{\Sigma}_{k+1}^{0}$-set), then the same holds for $A$.

The statement of the corollary has to be read either including both brackets or without both of them.

\section{$7 \quad$ Realizer reducibility}

In this section we will introduce another reducibility for total functions on metric spaces, called realizer reducibility, which generalizes the previously discussed one. The Representation Theorem 6.1 enables us to extend the Completeness Theorem 5.5 to this new reducibility. 
Definition 7.1 (Realizer reducibility) Let $X, Y, U, V$ be computable metric spaces and consider functions $f: X \rightarrow Y$ and $g: U \rightarrow V$. We define

$$
f \preceq_{\mathrm{t}} g: \Longleftrightarrow f \delta_{X} \leqslant_{\mathrm{t}} g \delta_{U}
$$

and we say that $f$ is realizer reducible to $g$, if this holds. Analogously, we define $f \preceq_{\mathrm{c}} g$ with $\leqslant_{\mathrm{c}}$ instead of $\leqslant_{t}$. The corresponding equivalences $\approx_{t}$ and $\approx_{c}$ are defined straightforwardly.

Reflexivity and transitivity of $\preceq_{\mathrm{t}}$ and $\preceq_{\mathrm{c}}$ can be directly concluded from the corresponding properties of $\leqslant_{t}$ and $\leqslant_{c}$. The following definition which is due to $[24,25,26]$ extends the reducibility $\leqslant_{t}$ to sets of functions.

Definition 7.2 (Set reducibility) Let $X, Y, U, V$ be computable metric spaces, let $\mathcal{F}$ be a set of functions $F: X \rightarrow Y$ and let $\mathcal{G}$ be a set of functions $G: U \rightarrow V$. We define

$$
\mathcal{F} \leqslant_{\mathrm{t}} \mathcal{G}: \Longleftrightarrow(\exists A, B \text { computable })(\forall G \in \mathcal{G})(\exists F \in \mathcal{F})(\forall x \in \operatorname{dom}(F)) F(x)=A(x, G B(x)),
$$

where $A: \subseteq X \times V \rightarrow Y$ and $B: \subseteq X \rightarrow U$. Analogously, one can define $\leqslant_{c}$ with computable $A, B$.

Using this definition we can derive the following characterization of realizer reducibility which explains the name.

Proposition 7.3 (Realizer reducibility) Let $X, Y, U, V$ be computable metric spaces and let $f: X \rightarrow Y$ and $g: U \rightarrow V$ be functions. Then

$$
f \preceq_{\mathrm{c}} g \Longleftrightarrow\{F: F \vdash f\} \leqslant_{\mathrm{c}}\{G: G \vdash g\} .
$$

An analogous statement holds with respect to $\preceq_{\mathrm{t}}$ and $\leqslant_{\mathrm{t}}$.

Proof. We will just consider the computable case. The topological case can be treated analogously.

Let $f \preceq_{\mathrm{c}} g$. Then $f \delta_{X} \leqslant_{\mathrm{c}} g \delta_{Y}$ and there are computable functions $A^{\prime}: \subseteq \mathbb{N}^{\mathbb{N}} \times V \rightarrow Y$ and $B: \subseteq \mathbb{N}^{\mathbb{N}} \rightarrow \mathbb{N}^{\mathbb{N}}$ such that $f \delta_{X}(p)=A^{\prime}\left(p, g \delta_{U} B(p)\right)$ for all $p \in \operatorname{dom}\left(\delta_{X}\right)$. Thus, there is a computable function $A: \subseteq \mathbb{N}^{\mathbb{N}} \times \mathbb{N}^{\mathbb{N}} \rightarrow \mathbb{N}^{\mathbb{N}}$ such that $\delta_{Y} A(p, q)=A^{\prime}\left(p, \delta_{V}(q)\right)$ for all $(p, q) \in$ $\operatorname{dom}\left(A^{\prime} \circ\left(\mathrm{id} \times \delta_{Y}\right)\right)$. Now, let $G \vdash g$, i.e. $\delta_{V} G=g \delta_{U}$. Then we obtain

$$
f \delta_{X}(p)=A^{\prime}\left(p, \delta_{V} G B(p)\right)=\delta_{Y} A(p, G B(p)) .
$$

Thus, $F: \subseteq \mathbb{N}^{\mathbb{N}} \rightarrow \mathbb{N}^{\mathbb{N}}$ with $\operatorname{dom}(F):=\operatorname{dom}\left(\delta_{X}\right)$ and $F(p):=A(p, G B(p))$ is a realizer of $f$, i.e. $F \vdash f$. Altogether, this proves $\{F: F \vdash f\} \leqslant_{\mathrm{c}}\{G: G \vdash g\}$.

Now let $\{F: F \vdash f\} \leqslant_{\mathrm{c}}\{G: G \vdash g\}$ and consider computable functions $A: \subseteq \mathbb{N}^{\mathbb{N}} \times \mathbb{N}^{\mathbb{N}} \rightarrow \mathbb{N}^{\mathbb{N}}$ and $B: \subseteq \mathbb{N}^{\mathbb{N}} \rightarrow \mathbb{N}^{\mathbb{N}}$ such that $(\forall G \vdash g)(\exists F \vdash f)(\forall p \in \operatorname{dom}(F)) F(p)=A(p, G B(p))$. This especially implies

$$
(\forall G \vdash g)\left(\forall p \in \operatorname{dom}\left(\delta_{X}\right)\right) f \delta_{X}(p)=\delta_{Y} A(p, G B(p)) .
$$

Let $R: V \rightarrow \mathbb{N}^{\mathbb{N}}$ be some right inverse of $\delta_{Y}$ and let $A^{\prime}: \subseteq \mathbb{N}^{\mathbb{N}} \times V \rightarrow Y$ be defined by $A^{\prime}(p, v)=\delta_{Y} A(p, R(v))$ for all $p \in \operatorname{dom}\left(\delta_{X}\right)=: D$ and $v=v_{p}:=g \circ \delta_{U} \circ B(p)$ (and undefined for all other $(p, v))$. Since $(3)$ holds for all $G \vdash g$, we obtain

$$
A^{\prime}\left(p, \delta_{V}(q)\right)=\delta_{Y} A\left(p, R \circ \delta_{V}(q)\right)=\delta_{Y} A(p, q)
$$


for all $p \in D$ and $q \in \delta_{V}^{-1}\left\{v_{p}\right\}$. Thus $A^{\prime}$ is computable. Now let us choose some $G \vdash g$ and define $G^{\prime}:=R \circ \delta_{V} \circ G$. Then $\delta_{V} G^{\prime}=\delta_{V} G=g \delta_{U}$ and thus $G^{\prime} \vdash g$ and we obtain by (3)

$$
f \delta_{X}(p)=\delta_{Y} A\left(p, G^{\prime} B(p)\right)=\delta_{Y} A\left(p, R \circ \delta_{V} \circ G B(p)\right)=A^{\prime}\left(p, \delta_{V} G \circ B(p)\right)
$$

for all $p \in \operatorname{dom}\left(\delta_{X}\right)=D$. Hence $f \delta_{X} \leqslant_{\mathrm{c}} \delta_{V} G=g \delta_{U}$ and thus $f \preceq_{\mathrm{c}} g$.

On Baire space the realizer reducibility does not yield anything new as the following lemma states.

Lemma 7.4 For functions $F, G: \mathbb{N}^{\mathbb{N}} \rightarrow \mathbb{N}^{\mathbb{N}}$ we obtain

(1) $F \preceq_{\mathrm{t}} G \Longleftrightarrow F \leqslant_{\mathrm{t}} G$,

(2) $F \preceq_{\mathrm{c}} G \Longleftrightarrow F \leqslant_{\mathrm{c}} G$.

This follows directly since the Cauchy representation $\delta_{\mathbb{N}^{\mathbb{N}}}$ is computable and it admits a computable right inverse function $\delta_{\mathbb{N N}}^{-}: \mathbb{N}^{\mathbb{N}} \rightarrow \mathbb{N}^{\mathbb{N}}$ with $\delta_{\mathbb{N}^{\mathbb{N}}} \circ \delta_{\mathbb{N}^{N}}^{-}=\operatorname{id}_{\mathbb{N}^{N}}$. The next proposition shows that $\preceq_{\mathrm{t}}$ and $\preceq_{\mathrm{c}}$ preserve $\boldsymbol{\Sigma}_{k}^{0}$-measurability and $\boldsymbol{\Sigma}_{k}^{0}$-computability, respectively. The proof of this property is based on the Representation Theorem 6.1 and thus, indirectly, on the effective Bhattacharya-Srivastava Selection Theorem 4.7.

Proposition 7.5 Let $X, Y, U, V$ be computable metric spaces and consider functions $f: X \rightarrow Y$ and $g: U \rightarrow V$. Then the following holds for all $k \geq 1$ :

(1) $f \preceq_{\mathrm{t}} g$ and $g$ is $\boldsymbol{\Sigma}_{k}^{0}$-measurable $\Longrightarrow f$ is $\boldsymbol{\Sigma}_{k}^{0}$-measurable,

(2) $f \preceq_{\mathrm{c}} g$ and $g$ is $\boldsymbol{\Sigma}_{k}^{0}$-computable $\Longrightarrow f$ is $\boldsymbol{\Sigma}_{k}^{0}$-computable.

Proof. We consider the computable case (2), the topological case can be proved analogously. If $f \preceq_{\mathrm{c}} g$ and $g$ is $\boldsymbol{\Sigma}_{k}^{0}$-computable, then we obtain $f \delta_{X} \leqslant_{\mathrm{c}} g \delta_{U}$ and since $\delta_{U}$ is computable it follows by Proposition 3.8 that $g \circ \delta_{U}$ is $\boldsymbol{\Sigma}_{k}^{0}$-computable. By Proposition 5.2 we obtain that $f \circ \delta_{X}$ is $\boldsymbol{\Sigma}_{k}^{0}$-computable. Thus, by the Representation Theorem 6.1 we can conclude that there is a $\boldsymbol{\Sigma}_{k}^{0}$-computable realizer $F$ of $f \circ \delta_{X}$. Thus, $f$ admits a $\boldsymbol{\Sigma}_{k}^{0}$-computable realizer since $\delta_{\mathbb{N}^{\mathbb{N}}}$ has a computable right inverse. It follows by applying the Representation Theorem 6.1 again that $f$ itself is $\Sigma_{k}^{0}$-computable.

By the following result we transfer the Completeness Theorem 5.5 to arbitrary computable metric spaces and realizer reducibility.

Theorem 7.6 (Completeness) Let $X, Y$ be computable metric spaces and let $k \in \mathbb{N}$. For any function $f: X \rightarrow Y$ we obtain:

(1) $f \preceq_{\mathrm{t}} C_{k} \Longleftrightarrow f$ is $\boldsymbol{\Sigma}_{k+1}^{0}-$ measurable,

(2) $f \preceq_{\mathrm{c}} C_{k} \Longleftrightarrow f$ is $\boldsymbol{\Sigma}_{k+1}^{0}$-computable.

Proof. We consider the computable case (2), the topological case (1) can be proved analogously. Let $f$ be $\boldsymbol{\Sigma}_{k+1}^{0}$-computable. Then by the Representation Theorem $6.1 f$ admits a $\boldsymbol{\Sigma}_{k+1}^{0}$-computable realizer $F$ and hence $F \leqslant_{\mathrm{c}} C_{k}$ by the Completeness Theorem 5.5. Since $\delta_{Y}$ is computable and $\delta_{\mathbb{N}^{\mathbb{N}}}$ admits a computable right inverse, it follows $f \delta_{X}=\delta_{Y} F \leqslant_{\mathrm{c}} C_{k} \delta_{\mathbb{N}^{\mathbb{N}}}$ and thus $f \preceq_{\mathrm{c}} C_{k}$. Now let, on the other hand, $f \preceq_{\mathrm{c}} C_{k}$. Since $C_{k}$ is $\Sigma_{k+1}^{0}$-computable by Theorem 5.5 , it follows that $f$ is $\boldsymbol{\Sigma}_{k+1}^{0}$-computable by Proposition 7.5. 
In the spirit of Corollary 5.8 and 6.6 we could formulate an effective version of the previous Completeness Theorem. We leave this to the reader. The Completeness Theorem gives rise to the following definition.

Definition 7.7 (Completeness) Let $X, Y$ be computable metric spaces, let $f: X \rightarrow Y$ be a function and $k \in \mathbb{N}$. Then $f$ is called $\boldsymbol{\Sigma}_{k+1}^{0}$ complete, if $f \approx_{c} C_{k}$.

In order to prove that a function $f: X \rightarrow Y$ is $\boldsymbol{\Sigma}_{k+1}^{0}$-complete, it suffices to show that it is $\boldsymbol{\Sigma}_{k+1}^{0}$-computable and that $C_{k} \leqslant_{c} f$. The $\boldsymbol{\Sigma}_{1}^{0}$-complete functions are exactly the computable functions $f: X \rightarrow Y$. Usually, it is harder to establish the lower bounds and therefore it is helpful to have methods in order to obtain these lower bounds. In [2] we have investigated such methods for the case $k=1$. One result was that the assumptions of the First Main Theorem of Pour-El and Richards [18] suffice to conclude that $C_{1} \leqslant_{c} f$ holds. For completeness, we formulate this result here again.

Theorem 7.8 (Lower bounds for unbounded linear closed operators) Let $X, Y$ be computable Banach spaces and let $f: \subseteq X \rightarrow Y$ be a closed linear and unbounded operator. Let $\left(e_{n}\right)_{n \in \mathbb{N}}$ be a computable sequence in $\operatorname{dom}(f)$ whose linear span is dense in $X$ and let $f\left(e_{n}\right)_{n \in \mathbb{N}}$ be computable in $Y$. Then $C_{1} \leqslant_{\mathrm{c}} f$.

This theorem can often be applied to derive that certain operators are $\boldsymbol{\Sigma}_{2}^{0}$-complete.

\section{Arithmetic complexity of points}

It is obvious that any computable function maps computable inputs to computable outputs. We would like to generalize this result to inputs of a certain level of the arithmetic hierarchy. Moreover, we have already seen in [2] that any function $f: \subseteq X \rightarrow Y$ with $C_{1} \leqslant \mathrm{c} f$ has a computable input $x \in X$ which is mapped to a non-computable output $f(x) \in Y$. We would like to generalize this idea as well and consider points of $\boldsymbol{\Sigma}_{k}^{0}$-complete functions. In the following we use the light face classes $\Sigma_{n}^{0}, \Pi_{n}^{0}$ and $\Delta_{n}^{0}:=\Sigma_{n}^{0} \cap \Pi_{n}^{0}$ to denote the arithmetical hierarchy [17] (thus, $\Sigma_{k}^{0}$ is the class of all computable sets in $\Sigma_{k}^{0}(\mathbb{N})$ etc.; in particular $\Sigma_{1}^{0}$ is the set of all r.e. subsets and $\Delta_{1}^{0}$ the set of all recursive subsets $A \subseteq \mathbb{N}$ ). A point $p \in \mathbb{N}^{\mathbb{N}}$ is called $\Delta_{n}^{0}$-computable, if $\operatorname{graph}(p)=\{\langle m, k\rangle \in \mathbb{N}: p(m)=k\} \in \Delta_{n}^{0}$. Thus, the $\Delta_{1}^{0}$-computable points $p \in \mathbb{N}^{\mathbb{N}}$ are just the ordinary computable points.

Definition 8.1 (Complexity of points) Let $X$ be a computable metric space and let $x \in X$. Then $x$ is called $\Delta_{n}^{0}$-computable, if there is a $\Delta_{n}^{0}$-computable $p \in \mathbb{N}^{\mathbb{N}}$ such that $x=\delta_{X}(p)$.

The arithmetical hierarchy of real numbers has been studied in [28]. See also [10] for related results.

We mention that for all $p, q \in \mathbb{N}^{\mathbb{N}}$ we have $\operatorname{graph}(q) \leq_{\mathrm{T}} \operatorname{graph}(p)$, if and only if there exists some computable $F: \subseteq \mathbb{N}^{\mathbb{N}} \rightarrow \mathbb{N}^{\mathbb{N}}$ such that $F(p)=q$ (here $\leq_{\mathrm{T}}$ denotes Turing reducibility [17]). Moreover, we note that whenever $A \leq_{\mathrm{T}} B$ and $B \in \Delta_{n}^{0}$, then $A \in \Delta_{n}^{0}$ follows for any $n \geq 1$ (this is because $A \in \Delta_{n}^{0}$, if and only if $A \leq_{\mathrm{T}} \emptyset^{(n-1)}$, where $\emptyset^{(n)}$ denotes the $n$-th Turing jump, see Post's Theorem IV.1.14 in [17]). Now we can formulate the following characterization of $\Delta_{n}^{0}$-computable points. 
Proposition 8.2 If $(X, d, \alpha)$ is a computable metric space such that the equivalence problem for balls $\{\langle m, k, i, j\rangle \in \mathbb{N}: B(\alpha(m), \bar{i})=B(\alpha(k), \bar{j})\}$ is r.e., then we obtain for any point $x \in X$ and $n \geq 1$ :

$$
x \text { is } \Delta_{n}^{0} \text {-computable } \Longleftrightarrow\{\langle m, i\rangle \in \mathbb{N}: x \in B(\alpha(m), \bar{i})\} \in \Sigma_{n}^{0} .
$$

This follows immediately from Theorem 8.1.4.2 in [26] and from the fact that $A \in \Sigma_{n}^{0}$ holds if and only if there exists some $p \in \mathbb{N}^{\mathbb{N}}$ such that $\operatorname{graph}(p) \in \Delta_{n}^{0}$ and range $(p)=A$, unless $A$ is empty. Now we want to study the behaviour of $\boldsymbol{\Sigma}_{k}^{0}$-computable functions on computable points. We will essentially use the Completeness Theorem 5.5 and the Representation Theorem 6.1 in order to prove the result.

Theorem 8.3 (Invariance Theorem) Let $X, Y$ be computable metric spaces. If $f: X \rightarrow Y$ is $\boldsymbol{\Sigma}_{k}^{0}$-computable, then it maps $\Delta_{n}^{0}$-computable inputs $x \in X$ to $\Delta_{n+k-1}^{0}$-computable outputs $f(x) \in Y$ for all $n, k \geq 1$. If $f$ is even $\boldsymbol{\Sigma}_{k}^{0}$-complete and $k \geq 2$, then there is some $\Delta_{n}^{0}$-computable input $x \in X$ for any $n \geq 1$ which is mapped to some $\Delta_{n+k-1}^{0}$-computable output $f(x) \in Y$ which is not $\Delta_{n+k-2}^{0}$-computable.

Proof. Let $f: X \rightarrow Y$ be $\Sigma_{k}^{0}$-computable for some $k \geq 1$. Then by the Representation Theorem $6.1 f$ admits a $\boldsymbol{\Sigma}_{k}^{0}$-computable realizer $F: D \rightarrow \mathbb{N}^{\mathbb{N}}$ with $D:=\operatorname{dom}\left(\delta_{X}\right)$. In case $k=1$ it is clear that $F$ maps $\Delta_{n}^{0}$-computable inputs $p \in D \subseteq \mathbb{N}^{\mathbb{N}}$ to $\Delta_{n}^{0}$-computable outputs $F(p)$ and thus $f$ has the desired property. Now let us assume that $k \geq 2$. Then $F \leqslant_{\mathrm{c}} C_{k-1}$ by the Completeness Theorem 5.5. Thus, there are computable functions $A, B: \subseteq \mathbb{N}^{\mathbb{N}} \rightarrow \mathbb{N}^{\mathbb{N}}$ such that $F(p)=A \circ C_{k-1} \circ B(p)$ for all $p \in D$. Since $A, B$ preserve the complexity of points, it suffices to prove the statement for $C_{k-1}$. By the Factorization Lemma 5.9 we can proceed inductively and it suffices to treat the case $k=2$. In this case $\operatorname{graph}(p) \in \Delta_{n}^{0}$ implies

$$
\begin{aligned}
\operatorname{graph}\left(C_{1}(p)\right) & =\{\langle l, m\rangle:(m=0 \text { and }(\exists k) p\langle l, k\rangle \neq 0) \text { or }(m=1 \text { and }(\forall k) p\langle l, k\rangle=0)\} \\
& \in\left\{A \cup B: A \in \Sigma_{n}^{0}, B \in \Pi_{n}^{0}\right\} \subseteq \Delta_{n+1}^{0} .
\end{aligned}
$$

Altogether this shows that any $\boldsymbol{\Sigma}_{k}^{0}$-computable $f: X \rightarrow Y$ maps $\Delta_{n}^{0}$-computable inputs $x \in X$ to $\Delta_{n+k-1}^{0}$-computable outputs $f(x) \in Y$ for all $n, k \geq 1$.

Now, let us assume that $f$ is even $\boldsymbol{\Sigma}_{k}^{0}$-complete for some $k \geq 2$. Then, by the Representation Theorem $6.1 f$ has some $\boldsymbol{\Sigma}_{k}^{0}$-computable realizer $F$ and $C_{k-1} \leqslant c f \delta_{X}=\delta_{Y} F$ holds in this case. Thus, there are computable maps $A: \subseteq \mathbb{N}^{\mathbb{N}} \times Y \rightarrow \mathbb{N}^{\mathbb{N}}$ and $B: \mathbb{N}^{\mathbb{N}} \rightarrow \mathbb{N}^{\mathbb{N}}$ such that $C_{k-1}(p)=$ $A\left(p, \delta_{Y} F B(p)\right)$ for all $p \in \mathbb{N}^{\mathbb{N}}$. Let us assume that we have already proved the statement for $C_{k-1}$, i.e. there is some $\Delta_{n}^{0}$-computable $p \in \mathbb{N}^{\mathbb{N}}$ such that $C_{k-1}(p)$ is not $\Delta_{n+k-2}^{0}$-computable. Since $A$ preserves the quality of inputs, it follows that $\delta_{Y} F B(p)$ has to be $\Delta_{n+k-1}^{0}-$ computable but not $\Delta_{n+k-2}^{0}$-computable. Since $B$ preserves the quality of inputs as well, it follows $x:=$ $\delta_{X} B(p) \in X$ is a $\Delta_{n}^{0}$-computable point with the property that $f(x)=\delta_{Y} F B(p) \in Y$ is $\Delta_{n+k-1^{-}}^{0}$ computable but not $\Delta_{n+k-2}^{0}$-computable. It remains to prove the statement for $C_{k-1}$. Since $\emptyset(n+k-2) \in \Sigma_{n+k-2}^{0}$, it follows by the Tarski-Kuratowski Normal Form Theorem IV.1.5 in [17] that there is some $A \in \Delta_{n}^{0}$ such that $\emptyset^{(n+k-2)}=\left\{m:\left(\exists n_{k-1}\right)\left(\forall n_{k-2}\right) \ldots\left\langle m, n_{1}, \ldots, n_{k-1}\right\rangle \in A\right\}$. Let $p$ be defined by

$$
p(m):=\left\{\begin{array}{ll}
1 & \text { if } m \in A \\
0 & \text { otherwise }
\end{array} .\right.
$$

Since $A \in \Delta_{n}^{0}$, it follows that $p$ is $\Delta_{n}^{0}$-computable. Since $\left(C_{k-1}(p)\right)^{-1}\{0\}=\emptyset^{(n+k-2)}$, it follows that $C_{k-1}(p)$ cannot be $\Delta_{n+k-2}^{0}$-computable. 
This result allows to conclude some obvious elementary facts on the reducibility of functions. In the following definition we define some "small" counterparts $c_{k}$ of the functions $C_{k}$.

Definition 8.4 For any $k \in \mathbb{N}$ we define $c_{k}: \mathbb{N}^{\mathbb{N}} \rightarrow \mathbb{N}^{\mathbb{N}}$ by

$$
c_{k}(p):= \begin{cases}0 & \text { if }\left(\exists n_{k}\right)\left(\forall n_{k-1}\right) \ldots p\left\langle n_{1}, \ldots, n_{k}\right\rangle \neq 0 \\ 1 & \text { otherwise }\end{cases}
$$

for all $p \in \mathbb{N}^{\mathbb{N}}$.

It is obvious that $c_{k}$ is $\boldsymbol{\Sigma}_{k+1}^{0}-$ computable for any $k \in \mathbb{N}$. The functions $c_{k}$ are characteristic functions of complete sets of the Wadge hierarchy $[15,11,26]$. It is known that one obtains $\mathrm{cf}_{A} \leqslant_{\mathrm{t}} \mathrm{cf}_{B} \Longleftrightarrow A \oplus A^{c} \leq_{\mathrm{W}} B \oplus B^{c}$ for all $A, B \subseteq \mathbb{N}^{\mathbb{N}}$ (see [26], here $\leq_{\mathrm{W}}$ denotes the Wadge reducibility). Since range $\left(c_{k}\right)$ contains only computable points, we can easily deduce the following facts from the Invariance Theorem 8.3. Here $f<_{\mathrm{c}} g$ means that $f \leqslant_{\mathrm{c}} g$ but $g \not_{\mathrm{c}} f$.

Proposition 8.5 We obtain for any $k \in \mathbb{N}$ :

(1) $C_{1} \mathbb{\mathrm { c }}_{\mathrm{c}} c_{k+1}<_{\mathrm{c}} C_{k+1}$,

(2) $C_{k}<_{\mathrm{c}}\left(C_{k}, c_{k+1}\right)<_{\mathrm{c}} C_{k+1}$.

The topological counterparts of these results require slightly different proofs (considering preimages). Here, this is just understood as a demonstration how complexity properties of points with respect to $\boldsymbol{\Sigma}_{k}^{0}$-computable functions can be employed. It is worth noticing that the functions $c_{k}$ correspond to LPO-principles in constructive mathematics [25]. We suggest to call a function $f: X \rightarrow Y \Sigma_{k}^{0}$-subcomplete, if $f \approx_{c} c_{k+1}$ holds.

\section{Effective Baire classification}

In this section we want to extend the classical Baire characterization of Borel measurable functions (see $[15,11]$ ) of certain levels to the effectively Borel measurable functions. As a preparation we discuss the limit map.

Proposition 9.1 (Limits) Let $X$ be a computable metric space. Then the space of convergent sequences $c:=\left\{\left(x_{n}\right)_{n \in \mathbb{N}} \in X^{\mathbb{N}}:\left(x_{n}\right)_{n \in \mathbb{N}} \in X^{\mathbb{N}}\right.$ converges $\}$ is a computable metric subspace of $X^{\mathbb{N}}$. The ordinary limit map

$$
\lim : c \rightarrow X,\left(x_{n}\right)_{n \in \mathbb{N}} \mapsto \lim _{n \rightarrow \infty} x_{n}
$$

is $\boldsymbol{\Sigma}_{2}^{0}$-computable and it is $\boldsymbol{\Sigma}_{2}^{0}$-complete, if there is a computable embedding $\iota:\{0,1\}^{\mathbb{N}} \hookrightarrow X$.

Proof. On the one hand, $\boldsymbol{\Sigma}_{2}^{0}$-computability follows by Proposition 3.2 and Lemma 3.3 from

$$
\lim ^{-1}(B(x, r))=\left(\bigcup_{n=0}^{\infty} X^{n} \times \bar{B}\left(x, r-2^{-n}\right)^{\mathbb{N}}\right) \cap c \in \boldsymbol{\Sigma}_{2}^{0}(c)
$$


and on the other hand, if there is a computable embedding $\iota:\{0,1\}^{\mathbb{N}} \hookrightarrow X$ and $\lim _{\{0,1\}^{\mathbb{N}}}$ denotes the limit map of space $\{0,1\}^{\mathbb{N}}$, then one shows $C_{1} \leqslant_{c} \lim _{\{0,1\}^{\mathbb{N}}} \leqslant_{c} \lim _{X}$. The first reduction follows with the help of the function $G: \mathbb{N}^{\mathbb{N}} \rightarrow\left(\{0,1\}^{\mathbb{N}}\right)^{\mathbb{N}}$ which is defined by $G(p)=\left(G_{m}(p)\right)_{m \in \mathbb{N}}$ where

$$
G_{m}(p)(n):= \begin{cases}0 & \text { if }(\exists k \leq m) p\langle n, k\rangle \neq 0 \\ 1 & \text { else }\end{cases}
$$

for any $p \in \mathbb{N}^{\mathbb{N}}$ and $m \in \mathbb{N}$. Obviously, $G$ is computable and $C_{1}(p)=\lim _{\{0,1\}^{\mathbb{N}}} G(p)$. The second reduction follows since $\iota$ is continuous and thus $\lim _{n \rightarrow \infty} \iota\left(p_{n}\right)=\iota\left(\lim _{n \rightarrow \infty} p_{n}\right)$ whenever $\left(p_{n}\right)_{n \in \mathbb{N}}$ is a convergent sequence in $\{0,1\}^{\mathbb{N}}$ and hence $\lim _{n \rightarrow \infty} p_{n}=\iota^{-1} \lim _{n \rightarrow \infty} \iota\left(p_{n}\right)$.

In general one cannot expect that the limit map is $\boldsymbol{\Sigma}_{2}^{0}$-complete; for instance for the one point space it is even computable (for the two point space $\{0,1\}$ it is neither $\boldsymbol{\Sigma}_{1}^{0}$-computable nor $\boldsymbol{\Sigma}_{2}^{0}-$ complete; the latter follows from the Invariance Theorem 8.3). Analogously to Proposition 3.10 we can now formulate a result on pointwise convergence. We omit the almost identical proof.

Proposition 9.2 (Pointwise convergence) Let $X, Y$ be computable metric spaces and $k \geq 1$. The following operation is computable:

$$
\lim : \subseteq \boldsymbol{\Sigma}_{k}^{0}(X \rightarrow Y)^{\mathbb{N}} \rightarrow \boldsymbol{\Sigma}_{k+1}^{0}(X \rightarrow Y),\left(f_{n}\right)_{n \in \mathbb{N}} \mapsto \lim _{n \rightarrow \infty} f_{n},
$$

defined for all sequences $\left(f_{n}\right)_{n \in \mathbb{N}}$ of $\boldsymbol{\Sigma}_{k}^{0}$-measurable functions $f_{n}: X \rightarrow Y$ which are pointwise convergent.

The reader should notice that we have not considered sequences of multi-valued operations as in Proposition 3.10 since the composition of a $\boldsymbol{\Sigma}_{2}^{0}$-computable function with a $\boldsymbol{\Sigma}_{k}^{0}$ computable multi-valued operation is not necessarily $\Sigma_{k+1}^{0}$-computable. We directly formulate a non-uniform corollary.

Corollary 9.3 Let $X, Y$ be computable metric spaces and $k \geq 1$. If $\left(f_{n}\right)_{n \in \mathbb{N}}$ is a computable and pointwise convergent sequence of $\boldsymbol{\Sigma}_{k}^{0}$-computable functions $f_{n}: X \rightarrow Y$, then the limit function $f: X \rightarrow Y$ is $\boldsymbol{\Sigma}_{k+1}^{0}$-computable.

We demonstrate that the presented methods can easily be employed to classify the complexity of certain operators (the following result has essentially been proved directly by von Stein [22]).

Proposition 9.4 (Differentiation) $\operatorname{Let}^{(1)}[0,1]$ be the computable metric subspace of $\mathcal{C}[0,1]$ which contains the continuously differentiable functions $f:[0,1] \rightarrow \mathbb{R}$. The operator of differentiation

$$
d: \mathcal{C}^{(1)}[0,1] \rightarrow \mathcal{C}[0,1], f \mapsto f^{\prime}
$$

is $\boldsymbol{\Sigma}_{2}^{0}$-complete.

Proof. It is known that $d$ is a linear closed an unbounded operator and it is easy to see that $d$ is computable on the dense sequence of rational polynomials. Hence, $C_{1} \leqslant_{\mathrm{c}} d$ follows easily by applying Theorem 7.8. On the other hand, we obtain

$$
f^{\prime}(x)=\lim _{n \rightarrow \infty} \frac{f\left(x+(1-x) 2^{-n}\right)-f\left(x-x 2^{-n}\right)}{2^{-n}}
$$


for all $f \in \mathcal{C}^{(1)}[0,1]$ and $x \in[0,1]$. Thus, $d$ can be obtained as a limit of a pointwise convergent sequence of $\boldsymbol{\Sigma}_{1}^{0}$-computable functions and is therefore $\boldsymbol{\Sigma}_{2}^{0}$-computable.

By the Invariance Theorem 8.3 the previous proposition implies the well-known result that there exists a computable function which has a continuous but non-computable derivative. Under certain assumptions we can even prove a certain inverse statement of Proposition 9.2.

Theorem 9.5 (Limit normal form) Let $X$ and $Y$ be computable metric spaces and let $k \geq 1$. There is a computable operation $\Lambda: \Sigma_{k+1}^{0}(X \rightarrow Y) \rightrightarrows \Sigma_{k}^{0}\left(X \rightrightarrows Y^{\mathbb{N}}\right)$ such that $\lim \circ L=f$ for all $f \in \boldsymbol{\Sigma}_{k+1}^{0}(X \rightarrow Y)$ and $L \in \Lambda(f)$.

Proof. Let $f \in \boldsymbol{\Sigma}_{k+1}^{0}(X \rightarrow Y)$ be given. Then by the Representation Theorem 6.1 we can effectively find some realizer $F \in \boldsymbol{\Sigma}_{k+1}^{0}\left(D \rightarrow \mathbb{N}^{\mathbb{N}}\right)$ of $f$ with $D:=\operatorname{dom}\left(\delta_{X}\right)$ and by the effective Completeness Theorem, i.e. Corollary 5.8, we can effectively find some computable functions $A, B: \subseteq \mathbb{N}^{\mathbb{N}} \rightarrow \mathbb{N}^{\mathbb{N}}$ with $F(p)=A \circ C_{k} \circ B(p)$ for all $p \in D$ and

$$
\operatorname{dom}(A)=\{\langle p, q\rangle:(\forall n)(((\exists m)(p\langle n, m\rangle=1)) \text { or }((\exists m)(q\langle n, m\rangle=1)))\} .
$$

Note that the function $A$ roughly corresponds to the function $A$ from the proof of Theorem 5.5 but it here it has been computably extended to a slightly larger domain. Now we obtain $C_{k}=C_{1} \circ F_{k-1}$ by the Factorization Lemma 5.9. Moreover, we can write $C_{1}=\lim _{\mathbb{N}^{\mathbb{N}}} G$ with the computable function $G: \mathbb{N}^{\mathbb{N}} \rightarrow\left(\{0,1\}^{\mathbb{N}}\right)^{\mathbb{N}}$ which is defined by $G(p)=\left(G_{m}(p)\right)_{m \in \mathbb{N}}$ where

$$
G_{m}(p)(n):= \begin{cases}0 & \text { if } n \leq m \text { and }(\exists k \leq m) p\langle n, k\rangle \neq 0 \\ 1 & \text { else }\end{cases}
$$

for any $p \in \mathbb{N}^{\mathbb{N}}$ and $m \in \mathbb{N}$ (similarly as in Proposition 9.2). The definition of $G$ guarantees that $\left(q_{m}\right)_{m \in \mathbb{N}} \in \operatorname{range}(G)$ implies $q_{m}(n)=1$ for almost all $n \in \mathbb{N}$ and thus $q_{m} \in \operatorname{dom}(A)$ for all $m \in \mathbb{N}$. This implies

$$
F(p)=A \circ C_{1} \circ F_{k-1} \circ B(p)=A \circ \lim _{\mathbb{N}^{\mathbb{N}}} \circ G \circ F_{k-1} \circ B(p)=\lim _{\mathbb{N}^{\mathbb{N}}} \circ A^{\mathbb{N}} \circ G \circ F_{k-1} \circ B(p)
$$

for all $p \in D$. Since $F_{k-1}$ is $\boldsymbol{\Sigma}_{k}^{0}$-computable, it follows that we can effectively determine $H:=A^{\mathbb{N}} \circ G \circ F_{k-1} \circ B \in \Sigma_{k}^{0}\left(D \rightrightarrows\left(\mathbb{N}^{\mathbb{N}}\right)^{\mathbb{N}}\right)$. Now one can show that there is a computable function $R: \subseteq\left(\mathbb{N}^{\mathbb{N}}\right)^{\mathbb{N}} \rightarrow \mathbb{N}^{\mathbb{N}}$ such that $\delta_{Y} \circ \lim _{\mathbb{N}^{\mathbb{N}}}=\lim _{Y} \circ \delta_{Y}^{\infty} \circ R$. Using this function we obtain

$$
f \delta_{X}=\delta_{Y} F=\delta_{Y} \lim _{\mathbb{N}^{\mathbb{N}}} \circ H=\lim _{Y} \circ \delta_{Y}^{\infty} \circ R \circ H .
$$

In case $k=1$ we can by Lemma 6.3 replace $\delta_{X}$ by $\sigma_{X}$ without loss of generality and thus we obtain a $\boldsymbol{\Sigma}_{1}^{0}$-measurable operation $L:=\delta_{Y}^{\infty} \circ R \circ H \circ \Delta_{X}: X \rightrightarrows Y^{\mathbb{N}}$ such that $f=\lim _{Y} \circ L$. In case $k>1$ we apply Proposition 6.4 and we effectively obtain a $\boldsymbol{\Sigma}_{k}^{0}$-computable $s: X \rightarrow \mathbb{N}^{\mathbb{N}}$ such that $\delta_{X} \circ s(x)=x$ and $L:=\delta_{Y}^{\infty} \circ R \circ H \circ s \in \Sigma_{k}^{0}(X \rightarrow Y)$ with $f=\lim _{Y} \circ L$.

As the proof shows, we can even obtain a single-valued $L$ in case of $k>1$. This does not hold true in case of $k=1$ in general as the space $Y=\{0,1\}$ shows (a $\boldsymbol{\Sigma}_{2}^{0}$-computable characteristic function cannot be obtained as the limit of continuous functions with binary image in general). In fact, the previous observation leads to a an effective version of the Banach-Hausdorff-Lebesgue Theorem (which follows from Proposition 3.8 using evaluation and sequencing). 
Corollary 9.6 (Effective Banach-Hausdorff-Lebesgue Theorem) Let $X$ and $Y$ be computable metric spaces and let $k \geq 2$. Then for any $\boldsymbol{\Sigma}_{k+1}^{0}$ computable function $f: X \rightarrow Y$ there is a computable sequence $\left(f_{n}\right)_{n \in \mathbb{N}}$ of $\boldsymbol{\Sigma}_{k}^{0}$-computable functions such that $f=\lim _{n \rightarrow \infty} f_{n}$. For $X=\mathbb{N}^{\mathbb{N}}$ this holds true in case $k=1$ as well.

It is known that in case $Y=\mathbb{R}$ at least the classical result can be extended to the case $k=1$ as well [11].

\section{Conclusions}

We have presented a number of results which allow to connect computable analysis to effective descriptive set theory. Since we are primarily interested in the finite levels of the Borel hierarchy, we have not studied any ordinal extensions of the presented results so far. However, in some cases it should be possible to extend the results in this direction.

\section{References}

[1] S. Bhattacharya and S. Srivastava, Selection theorems and invariance of Borel pointclasses, Proceedings of the American Mathematical Society 97 (1986) 707-711.

[2] V. Brattka, Computable invariance, Theoret. Comp. Sci. 210 (1999) 3-20.

[3] V. Brattka, Recursive and computable operations over topological structures, Informatik Berichte 255, FernUniversität Hagen, Fachbereich Informatik, Hagen, July 1999.

[4] V. Brattka, Computability of Banach space principles, Informatik Berichte 286, FernUniversität Hagen, Fachbereich Informatik, Hagen, June 2001.

[5] V. Brattka, Computability over topological structures, in: S. B. Cooper and S. S. Goncharov (eds.), Computability and Models, Kluwer Academic Publishers, New York 2003, 93-136.

[6] V. Brattka, Plottable real number functions and the computable graph theorem, Informatik Berichte 300, FernUniversität in Hagen, Fachbereich Informatik, Hagen, July 2003.

[7] V. Brattka and G. Presser, Computability on subsets of metric spaces, Theoret. Comp. Sci. 305 (2003) 43-76.

[8] A. Hemmerling, Approximate decidability in Euclidean spaces, Math. Log. Quart. 49 (2003) $34-56$.

[9] P. Hertling, Unstetigkeitsgrade von Funktionen in der effektiven Analysis, Informatik Berichte 208, FernUniversität Hagen, Hagen, Nov. 1996.

[10] C.-K. Ho, Relatively recursive reals and real functions, Theoret. Comp. Sci. 210 (1999) 99-120.

[11] A. S. Kechris, Classical Descriptive Set Theory, Springer, Berlin 1995.

[12] K.-I. Ko, Complexity Theory of Real Functions, Birkhäuser, Boston 1991. 
[13] C. Kreitz and K. Weihrauch, Theory of representations, Theoret. Comp. Sci. 38 (1985) $35-53$.

[14] K. Kuratowski and C. Ryll-Nardzewski, A general theorem on selectors, Bulletin de L'Académie Polonaise des Sciences 13 (1965) 397-403.

[15] Y. N. Moschovakis, Descriptive Set Theory, North-Holland, Amsterdam 1980.

[16] U. Mylatz, Vergleich unstetiger Funktionen in der Analysis, Diplomarbeit, Fachbereich Informatik, FernUniversität Hagen 1992.

[17] P. Odifreddi, Classical Recursion Theory, North-Holland, Amsterdam 1989.

[18] M. B. Pour-El and J. I. Richards, Computability in Analysis and Physics, Springer, Berlin 1989.

[19] J. Saint Raymond, Fonctions boréliennes sur un quotient, Bull. Sci. Math. (2) 100 (1976) $141-147$.

[20] M. Schröder, Topological spaces allowing type 2 complexity theory, in: K.-I. Ko and K. Weihrauch (eds.), Computability and Complexity in Analysis, vol. 190 of Informatik Berichte, FernUniversität Hagen, Sept. 1995, 41-53.

[21] M. Schröder, Extended admissibility, Theoret. Comp. Sci. 284 (2002) 519-538.

[22] T. v. Stein, Vergleich nicht konstruktiv lösbarer Probleme in der Analysis, Diplomarbeit, Fachbereich Informatik, FernUniversität Hagen 1989.

[23] K. Weihrauch, Computability, Springer, Berlin 1987.

[24] K. Weihrauch, The degrees of discontinuity of some translators between representations of the real numbers, Technical Report TR-92-050, International Computer Science Institute, Berkeley, July 1992.

[25] K. Weihrauch, The TTE-interpretation of three hierarchies of omniscience principles, Informatik Berichte 130, FernUniversität Hagen, Hagen, Sept. 1992.

[26] K. Weihrauch, Computable Analysis, Springer, Berlin 2000.

[27] K. Weihrauch, Computational complexity on computable metric spaces, Math. Log. Quart. 49 (2003) 3-21.

[28] X. Zheng and K. Weihrauch, The arithmetical hierarchy of real numbers, Math. Log. Quart. 47 (2001) 51-65. 